\title{
UV considerations on scattering amplitudes in a web of theories
}

\author{
John Joseph M. Carrasco ${ }^{1,2}$ and Laurentiu Rodina $\odot^{2}$ \\ ${ }^{1}$ Department of Physics and Astronomy, Northwestern University, Evanston, Illinois 60208, USA \\ ${ }^{2}$ Institut de Physique Theorique, Universite Paris Saclay, CEA, CNRS, F-91191 Gif-sur-Yvette, France
}

(Received 29 August 2019; published 6 December 2019)

\begin{abstract}
The scattering predictions of a web of theories including Yang-Mills, gravity, biadjoint scalar, the nonlinear sigma model (NLSM), Dirac-Born-Infeld-Volkov-Akulov, and the special Galileon (sGal) form a class of special objects with two fascinating properties: they are related by the double-copy procedure, and they can be defined purely by on-shell constraints. We expand on both of these properties. First we show that NLSM tree-level amplitudes are fully determined by imposing color-dual structure together with cyclic invariance and locality. We then consider how hard scaling can be used to constrain the predictions of these theories, as opposed to the usual soft scaling. We probe the UV by generalizing the familiar BrittoCachazo-Feng-Witten (BCFW) shift off-shell to a novel single hard limit. We show that UV scalings are sufficient to fully constrain (i) biadjoint doubly ordered amplitudes, assuming locality, (ii) the NLSM and the Born-Infeld theory, assuming locality and unitarity, and (iii) special Galileon theory, assuming locality, unitarity, and a UV bound for the general Galileon vertex. We see how potentially distinct aspects of this UV behavior can be understood and unified via double-copy relations. Surprisingly, we find evidence that assuming unitarity for these theories may not be necessary, and can emerge via UV considerations and locality alone. These results complete the observations that, like IR considerations, UV scaling is sufficient to fully constrain a wide range of tree-level amplitudes, for both gauge, gravity, and effective field theories.
\end{abstract}

DOI: 10.1103/PhysRevD.100.125007

\section{INTRODUCTION}

On-shell ideas and methods have transformed our approach to understanding perturbative predictions in relativistic quantum field theories. Doing so has exposed structure and relations between field theories from our most formal UV completions in string theory to the grittiest phenomenological theories living well in the IR, features completely hidden by off-shell Lagrangian formulations. Two such discoveries stand out and will be explored in this article:

(1) Various scattering amplitudes may be determined uniquely by on-shell principles

(2) These special scattering amplitudes form an intricate web of relations.

\section{A. On-shell consistency and uniqueness}

While it is a central tenet of quantum field theory that through Lagrangian symmetries determine an overwhelming majority of our known theories, it was only recently understood that some symmetries are even more

Published by the American Physical Society under the terms of the Creative Commons Attribution 4.0 International license. Further distribution of this work must maintain attribution to the author(s) and the published article's title, journal citation, and DOI. Funded by SCOAP ${ }^{3}$. constraining directly at the level of on-shell scattering amplitudes, which can avoid much of the complicated and redundant machinery of off-shell descriptions.

For instance, it is common knowledge that gauge invariance fixes the Lagrangian of gauge theories [like QED, Yang-Mills, but also general relativity (GR)], but in fact gauge invariance and locality alone are sufficient to fully fix amplitudes in Yang-Mills (YM) and GR, with unitarity emerging as a consequence [1,2]. Similarly, effective field theories (EFT's), long known to satisfy symmetries related to IR properties [3-7], were just recently seen to follow directly from the on-shell Adler zero condition $[1,2,8,9]$.

Yet interesting soft properties are not unique to EFT's: virtually all theories possess soft theorems, another very well-known fact, newly rediscovered and explored in many unexpected contexts [10-13]. In [14] the power of the IR was understood in a unified way: soft theorems are sufficient to fully constrain a large variety of theories, including both gauge and effective field theories. This lead to the surprising conclusion that the IR contains all the information needed to rebuild the amplitude — in other words, the IR somehow also knows about the UV pieces of amplitudes.

Closer to the UV probing of amplitudes, an orthogonal and very practical approach to the on-shell program has been the Britto-Cachazo-Feng-Witten (BCFW) recursion [15], which represented a milestone in the conceptual understanding and technical calculation of scattering 
amplitudes. Leveraging insight into the unitarity construction at loop level with Cauchy's theorem and amplitude UV behavior, it formed a concrete realization that for a special set of constructable theories, including Yang-Mills and gravity $[16,17]$, only minimal on-shell data-the threepoint amplitude-for these theories was required to completely specify all order predictions. This is in stark contrast with the requirement for higher-order contact terms in the action (infinitely many for GR), but which only exist to ensure gauge invariance. Effective field theories, on the other hand, in general have no such structure-without fundamental symmetries constraining higher orders, every contact term can have an arbitrary coefficient and so lowermultiplicity amplitudes are not enough to determine the higher-point ones. This is manifested directly in the presence of poles at infinity which obstruct the recursion, a reflection of the bad UV behavior of EFT's. However, the special theories under consideration do satisfy a symmetry which fixes the higher-point contact terms: the Adler zero, which in this sense can be regarded as a "gauge symmetry" for these scalar theories. Inspired by this observation, the $\mathrm{BCFW}$ recursion was extended to EFT's, by modifying the shifts as to include the Adler zero property [18-24].

Whether good or bad, the UV scaling is of central importance, but has often been viewed as an annoying obstacle which in some cases can be miraculously removed, thus allowing the recursion and computations to take place. A closer analysis of the scaling reveals that, like for gauge invariance or the Adler zero, numerous cancellations take place, suggesting that only very special objects can have this property, which may ultimately be related to a symmetry [25]. And indeed, in [26] it was argued that YM and GR treelevel amplitudes (along with their gauge invariance and unitarity) do in fact follow from locality and an improved UV scaling. But the UV scaling of EFT's, while not as good as that of gauge theories, is still highly improved over naive power counting based on Feynman rules.

Therefore, in this article we explore the UV properties of various tree-level EFT's, probed by two different deformations: a two line BCFW shift and a single hard limit, as opposed to the usual single soft limit. We will find that the nonlinear sigma model (NLSM) and Born-Infeld (BI), Galileon (Gal), and special Galileon (sGal) theories are all fixed by locality and demand some particular large $z$ scaling, bringing EFT UV considerations on the same footing as those of gauge theories. This is particularly surprising since it has long been thought that only IR information can be used to constrain EFT's, since they themselves live in the IR. And perhaps most surprisingly of all, we find that even biadjoint scalar amplitudes are fixed by such UV considerations. Since these amplitudes have trivial numerators, this example directly demonstrates that UV scaling is somehow probing unitarity.

We are then left with a puzzling fact, which yet lacks a complete explanation: both the IR (as expressed through the soft theorems) and the UV (probed through hard limits) are sufficient to fully fix a wide range of theories.

\section{B. A color-kinematic web of amplitude relations}

The other structure that appears to have a certain amount of ubiquity in S-matrix predictions, and relates theories discussed here, is the so-called color-dual double-copy structure, originally realized in Yang-Mills theory and its relation to gravity by Bern, Johansson, and one of the current authors (BCJ) $[27,28]$. This structure allows many amplitudes to be expressed as a generalized product between different building blocks, providing a purely field theoretic understanding and generalization of the celebrated Kawai-Lewellen-Tye (KLT) formula [29]:

$$
\text { gravity }=\text { Yang-Mills } \otimes \text { Yang-Mills }
$$

At the heart of this structure lies the color-kinematic duality, which schematically states that given an amplitude expressed in a color ordered amplitude basis, for example a tree-level YM amplitude,

$$
\mathcal{A}^{\mathrm{YM}}=\sum_{\sigma \in S_{n-2}} c_{\sigma} A^{\mathrm{YM}}(1, \sigma, n),
$$

there exist kinematic functions $n_{\sigma}^{\mathrm{YM}}$ that satisfy the same algebra as the color factors $c_{\sigma}$, and can replace them, producing the GR amplitude:

$$
\mathcal{A}^{\mathrm{GR}}=\sum_{\sigma \in S_{n-2}} n_{\sigma}^{\mathrm{YM}} A^{\mathrm{YM}}(1, \sigma, n) .
$$

The story goes deeper, as there exist other similar functions $n_{\sigma}^{\mathrm{NLSM}}$, which instead lead to an expression for the BI amplitudes:

$$
\mathcal{A}^{\mathrm{BI}}=\sum_{\sigma \in S_{n-2}} n_{\sigma}^{\mathrm{NLSM}} A^{\mathrm{YM}}(1, \sigma, n) .
$$

This structure is what leads to the KLT factorization for adjoint-compatible amplitudes, as well as the scattering equations allowing for the Cachazo-He-Yuan expression of tree-level amplitudes [30-35]. The web is even more tangled, as transmutation operators can directly transform some amplitudes into others [36,37]. Perhaps more intriguing even than such tree-level relations, these kinematic functions $n$ have a local interpretation relevant to a graph representation of the amplitudes-theory specific dressings dual to $f^{a b c}$ color weights, over scalar propagators. In essence, these functions have the same job as color-charges weights, locally dressing graphs with some kinematic function relevant to the building blocks of the theory at hand. This seamlessly generalizes to the multiloop corrections at the integrand level. With just a small set of these color-chargelike weights, $c, n^{\mathrm{YM}}, n^{\mathrm{NLSM}}$ obeying the same 
algebraic relations (namely Jacobi and antisymmetry about vertices), one can build full multiloop amplitudes for this family of theories:

$$
\mathcal{A}_{n}^{(L)} \propto \sum_{i} \int \frac{d^{L D} \ell}{(2 \pi)^{L D}} \frac{1}{S_{i}} \frac{m_{i} \tilde{m}_{i}}{D_{i}}
$$

where various theories are given by the choices of $(m, \tilde{m})$ given in Table I. In these cases of an adjoint double copy, the sums run over all distinct $L$-loop $m$-point cubic diagrams, the $S_{i}$ represent the symmetry factors of the graphs, and the $D_{i}$ are massless scalar propagators relevant to each graph.

The striking dual role between the kinematic and charge graph weights invites potentially fundamental as of yet unanswered questions. What does it mean that we can treat gravitons as gluons whose charge is the kinematics of gluons? What does it mean to think of Born-Infeld photons as gluons whose charge is the kinematics of pions? While the conceptual implications of a color-dual double-copy structure has yet to be fully realized, the technical impact of the resulting algebraic constraints has however already been far reaching. It allows perturbative calculations in quantum gravity to achieve loop orders previously believed to be entirely out of reach by reframing them as predictions of much more tractable quantum Yang-Mills calculations $[28,38,39]$. These ideas have since been extended far beyond their original domain of on-shell scattering amplitudes to include form factors $[40,41]$, analysis of symmetries in gravity theories [42-48], classical scalar, gauge, and gravity solutions [49-62], and very recent implications for GR wave calculations. Indeed the highest order postMinkowski corrections to classical binary black-hole dynamics to date (3PM) has been carried out by leveraging these fundamental structures to fix coefficients in an effective action [63,64].

It is not clear what symmetry is responsible for this structure, nor the algebra the various kinematic graphical weights are charged under, not to mention the physical implications for the building blocks of fundamental theories. We therefore initiate an exploration of this last question, framing it in the context of on-shell constraints: What (local) objects can satisfy the color kinematic duality?
We discover that in certain cases this condition is more constraining than previously thought. Applying it to the simplest kinematic example, we find that it uniquely fixes the NLSM amplitudes and some of its higher derivative corrections.

It is also noteworthy that both open and closed string theory amplitudes at tree level manifest a field-theoretic adjoint color-dual double-copy structure. The fact that double copy seems compulsory to the effective building blocks of the only known ultraviolet completions of (higher-dimensional) Yang-Mills and gravity theories is incredibly tantalizing and suggests a compatibility with UV completion. This further motivates the UV exploration that we will carry out.

The paper is organized as follows. In Sec. II we briefly introduce the theories under consideration: biadjoint scalar, NLSM, Dirac-Born-Infeld-Volkov-Akulov (DBI-VA), Gal, and sGal. In Sec. III we describe the on-shell constraints which will be used: locality, unitarity, soft limits, and the UV probes: the two-particle BCFW shift, for both scalars and vectors, and the single hard limit, which can be understood as an off-shell BCFW shift. In Sec. IV we expand on the amplitude relations, and show that the NLSM is fixed by amplitude relations, locality, and mass dimension. In Sec. $\mathrm{V}$ we present several uniqueness conditions that follow from imposing UV constraints and mass dimension considerations:

(i) biadjoint doubly ordered amplitudes from locality,

(ii) NLSM and BI from locality and unitarity,

(iii) sGal from locality, unitarity, and a bound on the UV scaling of the Gal vertex.

We present evidence that uniqueness can still follow even after dropping the unitarity constraint. We summarize and discuss the outlook for future results in Sec. VI.

\section{FIELD THEORIES}

\section{A. Conventions}

In this article we elide both phases and coupling constants to minimize unnecessary clutter. An often used convention is to delegate coupling constants to full amplitudes (not ordered amplitudes), and set graph weights in accordance with the following double-copy prescription for full adjoint double-copy amplitudes as

TABLE I. Factorization to adjoint color-dual graph numerators for this double-copy web of theories.

\begin{tabular}{lcc}
\hline \hline Theory & $m$ & $\tilde{m}$ \\
\hline Biadjoint $\phi^{3}$ & $c\left(f^{a b c}\right)$ [color] & $\tilde{c}\left(\tilde{f}^{a b c}\right)$ (color) \\
Yang-Mills & $c\left(f^{a b c}\right)$ [color] & $n^{\mathrm{YM}}(k, \epsilon)$ [vector] \\
gravity + axion + dilaton & $n^{\mathrm{YM}}(k, \epsilon)$ [vector] & $n^{\mathrm{YM}}(k, \epsilon)$ [vector] \\
NLSM & $c\left(f^{a b c}\right)[$ flavor/color] & $n^{\mathrm{NLSM}}(k)$ [scalar] \\
Born-Infeld & $n^{\mathrm{YM}}(k, \epsilon)$ [vector] & $n^{\mathrm{NLSM}}(k)$ [scalar] \\
Special Galileon & $n^{\mathrm{NLSM}}(k)$ [scalar] & $n^{\mathrm{NLSM}}(k)$ [scalar] \\
\hline \hline
\end{tabular}




$$
i^{L+1} \mathcal{A}_{n}^{\mathrm{Dbl.cpy}(L)}=g_{m \otimes \tilde{m}}^{n-2+2 L} \sum_{i} \int \frac{d^{L D} \ell}{(2 \pi)^{L D}} \frac{1}{S_{i}} \frac{m_{i} \tilde{m}_{i}}{D_{i}},
$$

where the sum is over all cubic graphs of loop order $L$ and multiplicity $n$, both $m$ and $\tilde{m}$ obey adjoint color relations, namely Jacobi and antisymmetry, the $D_{i}$ are the massless propagators, $S_{i}$ are any symmetry factors of graph $i$, and $g_{m \otimes \tilde{m}}$ is the coupling constant for the theory. The doublecopy construction then specifies the necessary scaling of coupling constants, e.g., $g_{\mathrm{YM}}=g$ and $g_{\mathrm{GR}}=\kappa / 2$, with associated phases and factors of $\sqrt{2}$ in color factors and traces. A thorough treatment of such a convention is given in Ref. [65].

\section{B. Biadjoint $\phi^{3}$}

The biadjoint scalar theory (see e.g., $[32,55,66-71]$ and references therein) is the simplest theory within the web of amplitudes we are discussing, and provides the clearest formal access to fundamental structure at the heart of all the predictions within this web. It is a theory of scalars which carries two colors and has a simple $\phi^{3}$ interaction,

$$
\mathcal{L}=\frac{1}{2} \partial^{\mu} \Phi^{a a^{\prime}} \partial_{\mu} \Phi^{a a^{\prime}}+\frac{1}{3} f^{a b c} \tilde{f}^{a^{\prime} b^{\prime} c^{\prime}} \Phi^{a a^{\prime}} \Phi^{b b^{\prime}} \Phi^{c c^{\prime}}
$$

The full amplitudes can be decomposed into doubly ordered partial amplitudes,

$$
\begin{aligned}
\mathcal{A}= & \sum_{\sigma, \rho \in S_{n-1}} \operatorname{Tr}\left(T^{a_{1}} T^{a_{\sigma(2)}} \ldots T^{a_{\sigma(n)}}\right) \times A_{n}^{\mathrm{bi}-\mathrm{Adj}}(1, \sigma \mid 1, \rho) \\
& \times \operatorname{Tr}\left(\tilde{T}^{b_{1}} \tilde{T}^{b_{\rho(2)}} \ldots \tilde{T}^{b_{\rho(n)}}\right) \\
= & \sum_{\sigma, \rho \in S_{n-2}} c(1|\sigma| n) A_{n}^{\mathrm{bi}-\mathrm{Adj}}(1, \sigma, n \mid 1, \rho, n) \tilde{c}(1|\rho| n) .
\end{aligned}
$$

These doubly ordered partial amplitudes $A_{n}(\sigma \mid \rho)$ are not unique to any theory, rather as we will see they encode the compatibility of a cubic propagator structure with Jacobi satisfying graphical weights. Indeed the $c(\sigma), \tilde{c}(\rho)$ functions are simply the color weights given to the half-ladder graphs of permutation $\sigma$ and $\rho$ by dressing each vertex with the Lie-algebra structure constants $f^{a b c}, \tilde{f}^{a^{\prime} b^{\prime} c^{\prime}}$. Why is this sufficient? Jacobi relations express color-charges for the set of all distinct $(2 n-5) ! ! n$-point graphs in terms of a basis set of $(n-2)$ ! half-ladder (also called multiperipheral) graphs with the two farthest leg-labels fixed [72].

Introducing a propagator containing matrix $P$ defined to be the $(2 n-5) ! ! \times(2 n-5) ! !$ diagonal matrix defined as $P_{i j}=\delta_{i, j} \frac{1}{D_{i}}$ where each nonvanishing element is the product of propagators for a particular graph, the full amplitude can be written explicitly in terms of all graphs as

$$
\mathcal{A}=\sum_{i=1}^{(2 n-5) ! !} \frac{c_{i} \tilde{c}_{i}}{D_{i}}=c_{\mathrm{all}} \cdot P \cdot \tilde{c}_{\mathrm{all}} \cdot
$$

The Jacobi solution matrix $J$ matrix is a $(2 m-5) ! ! \times$ $(m-2)$ ! matrix encoding how every graph's color factor is expressed via Jacobi relations terms of a basis of $(m-2)$ ! master graph color factors: $\left(c_{\text {all }}\right)=J \cdot\left(c_{\text {masters }}\right)$. This makes it clear that the doubly ordered biadjoint amplitude can be written as

$$
A^{\mathrm{bi}-\mathrm{Adj}}=J^{T} \cdot P \cdot J,
$$

where the matrix indices of the doubly ordered amplitude

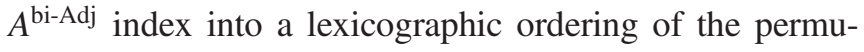
tations $\sigma$ and $\rho$-specifying what master graphs define $J$.

It is worth considering an example at four points with the following definitions for the three cubic color weights:

$$
\begin{gathered}
c_{s}=c(1|23| 4)=f^{a_{1} a_{2} b} f^{b a_{3} a_{4},} \\
c_{t}=f^{a_{4} a_{1} b} f^{b a_{2} a_{3},} \\
c_{u}=c(1|32| 4)=f^{a_{1} a_{3} b} f^{b a_{2} a_{4},}
\end{gathered}
$$

and $\tilde{c}_{i}=\left.c_{i}\right|_{f \rightarrow \tilde{f}}$, satisfying Jacobi: $c_{t}=c_{s}-c_{u}$ and $\tilde{c}_{t}=\tilde{c}_{s}-\tilde{c}_{u}$. The kinematic propagators are specified by Mandelstam variables, in an all outgoing convention:

$$
\begin{aligned}
& s=\left(k_{1}+k_{2}\right)^{2}=\left(k_{3}+k_{4}\right)^{2}, \\
& t=\left(k_{1}+k_{4}\right)^{2}=\left(k_{2}+k_{3}\right)^{2}, \\
& u=\left(k_{1}+k_{3}\right)^{2}=\left(k_{2}+k_{4}\right)^{2},
\end{aligned}
$$

satisfying $s+t+u=0$. So our full amplitude is given as

$$
\begin{aligned}
\mathcal{A}= & \frac{c_{s} \tilde{c}_{s}}{s}+\frac{c_{t} \tilde{c}_{t}}{t}+\frac{c_{u} \tilde{c}_{u}}{u} \\
= & {\left[\begin{array}{lll}
c_{s} & c_{t} & c_{u}
\end{array}\right] \cdot\left[\begin{array}{ccc}
\frac{1}{s} & 0 & 0 \\
0 & \frac{1}{t} & 0 \\
0 & 0 & \frac{1}{u}
\end{array}\right] \cdot\left[\begin{array}{c}
\tilde{c}_{s} \\
\tilde{c}_{t} \\
\tilde{c}_{u}
\end{array}\right] } \\
= & {\left[\begin{array}{ll}
c_{s} & c_{u}
\end{array}\right] \cdot\left[\begin{array}{ccc}
1 & 1 & 0 \\
0 & -1 & 1
\end{array}\right] \cdot\left[\begin{array}{ccc}
\frac{1}{s} & 0 & 0 \\
0 & \frac{1}{t} & 0 \\
0 & 0 & \frac{1}{u}
\end{array}\right] } \\
& \cdot\left[\begin{array}{cc}
1 & 0 \\
1 & -1 \\
0 & 1
\end{array}\right] \cdot\left[\begin{array}{c}
\tilde{c}_{s} \\
\tilde{c}_{u}
\end{array}\right]
\end{aligned}
$$




$$
\begin{aligned}
& =\left[\begin{array}{ll}
c_{s} & c_{u}
\end{array}\right] \cdot\left[\begin{array}{cc}
\frac{1}{s}+\frac{1}{t} & -\frac{1}{t} \\
-\frac{1}{t} & \frac{1}{t}+\frac{1}{u}
\end{array}\right] \cdot\left[\begin{array}{c}
\tilde{c}_{s} \\
\tilde{c}_{u}
\end{array}\right] \\
& =c_{\text {masters }}^{\mathrm{T}} \cdot A^{\mathrm{bi}-\mathrm{Adj}} \cdot \tilde{c}_{\text {masters }} .
\end{aligned}
$$

We will follow the convention in the literature and refer to these doubly ordered quantities as biadjoint amplitudes, but wish to emphasize that they are far more universal than biadjoint scalar $\phi^{3}$. Only when dressing with two color weights does the doubly ordered biadjoint amplitude build the biadjoint scalar amplitude. Every one of the amplitudes in the adjoint web of theories can be expressed by replacing these master graph color weights with color-dual kinematic weights $m$ or $\tilde{m}$ as per Table I,

$$
\mathcal{A}^{\text {Dbl.Copy }}=\sum_{\sigma, \rho \in S_{n-2}} m(1|\sigma| n) A^{\text {bi-Adj }}(1, \sigma, n \mid 1, \rho, n) \tilde{m}(1|\rho| n) \text {. }
$$

One can wonder if the doubly partial biadjoint amplitudes have a simple closed form expression, and indeed they do, given as the inverse of the KLT matrix [32,73] [cf. Eq. (111)]. This is simplest to see in the case of an $(n-3)$ ! basis, where the KLT matrix has a trivial inversion. There is however no real barrier to the $(n-2)$ ! symmetric KLT matrix, but there is a subtlety as its inversion requires regulation on shell. A procedure of inverting off shell, canceling the on-shell singularity with $\left(k_{n}\right)^{2}$, and only than taking the on-shell limit $\left(k_{n}\right)^{2} \rightarrow 0$ is similar to that discussed in e.g., Ref. [74] as it is intimately related to finding local color-dual kinematic weights in terms of ordered amplitudes using KLT. As the double adjoint striation collects trivalent graph propagators in terms of their dual-Jacobi master dressings, these objects are at the heart of adjoint-double copy.

In the simplest case when $\sigma=\rho$, the partial amplitude is simply the sum over the propagators of cubic graphs consistent with that color order:

$$
\begin{aligned}
A_{4}^{\text {bi-Adj }}(1,2,3,4 \mid 1,2,3,4)=\frac{1}{s_{12}}+\frac{1}{s_{14}}, & \\
A_{5}^{\text {bi-Adj }}(1,2,3,4,5 \mid 1,2,3,4,5)= & \frac{1}{s_{12} s_{34}}+\frac{1}{s_{23} s_{45}}+\frac{1}{s_{34} s_{51}} \\
& +\frac{1}{s_{45} s_{12}}+\frac{1}{s_{51} s_{23}} .
\end{aligned}
$$

In other cases, it is given by the set of propagators common to both orderings.

\section{NLSM}

The nonlinear sigma model [75-77] is a pionic theory of Nambu-Goldstone bosons, which arises from spontaneously breaking a Lie group $G \times G \rightarrow G$. It can be described by a Lagrangian in the Cayley parametrization [18,78-80],

$$
\mathcal{L}_{\mathrm{NLSM}}=\frac{1}{2} \operatorname{Tr}\left\{\partial_{\mu} \varphi \frac{1}{1-\lambda \varphi^{2}} \partial^{\mu} \varphi \frac{1}{1-\lambda \varphi^{2}}\right\}
$$

where $\varphi$ is a Lie-algebra-valued Goldstone-boson scalar field in the adjoint representation.

We will focus on the $S U(N)$ NLSM amplitudes $\mathcal{A}_{n}$, which can be decomposed into flavor-ordered "partial amplitudes" $A_{n}$ :

$$
\mathcal{A}_{n}=\sum_{\sigma \in S_{n-1}} \operatorname{Tr}\left(T^{a_{1}} T^{a_{\tau(2)}} \ldots T^{a_{\sigma(n)}}\right) A(1, \tau(2, \ldots, n)) .
$$

The four- and six-point ordered amplitudes read

$$
\begin{gathered}
A_{4}^{\mathrm{NLSM}}(1,2,3,4)=s_{13}, \\
A_{6}^{\mathrm{NLSM}}(1,2,3,4,5,6)=\frac{s_{13} s_{46}}{s_{123}}-s_{13}+(\text { cyclic }) .
\end{gathered}
$$

The Lagrangian enjoys a shift symmetry, equivalent to the Adler zero condition (see $[9,71]$ ), which in turn is sufficient to fully determine the on-shell amplitudes.

\section{DBI-VA}

We will also consider the Dirac-Born-Infeld-VolkovAkulov model. This is a nonlinear extension of Maxwell theory, which in $D$ dimensions is given by the following Lagrangian:

$$
\mathcal{L}_{\mathrm{BI}}=\sqrt{(-1)^{D-1} \operatorname{det}\left(\eta_{\mu \nu}+F_{\mu \nu}\right)} .
$$

Its supersymmetric extension has also been considered [81,82].

The scalar part, known as DBI, is fixed by a stronger Adler zero condition $\mathcal{O}\left(\tau^{2}\right)$, which similarly follows from a more general shift symmetry. Intriguingly, for the vector part, known as BI, in [83] it was shown that starting from a general Lagrangian

$$
\mathcal{L}_{\mathrm{BI}}=F^{2}+g_{4} F^{4}+g_{6} F^{6}+\ldots
$$

and demanding an improved low energy behavior, the coefficients $g_{i}$ can be fixed to match the expansion of (30). 4D amplitudes in the vector, fermion, and respectively scalar sector are given by

$$
A_{4}^{\mathrm{BI}}\left(\gamma_{1}^{-}, \gamma_{2}^{-}, \gamma_{3}^{+}, \gamma_{4}^{+}\right)=\langle 12\rangle^{2}[34]^{2},
$$

$A_{6}^{\mathrm{BI}}\left(\gamma_{1}^{-}, \gamma_{2}^{-}, \gamma_{3}^{-}, \gamma_{4}^{+}, \gamma_{5}^{+}, \gamma_{6}^{+}\right)=\frac{\langle 12\rangle^{2}[56]^{2}\langle 3|1,2| 4]^{2}}{s_{124}}+$ perms. 


$$
\begin{aligned}
& A_{4}^{\mathrm{VA}}\left(\psi_{1}, \psi_{2}, \bar{\psi}_{3}, \bar{\psi}_{4}\right)=\langle 12\rangle[34] s_{12}, \\
& A_{6}^{\mathrm{VA}}\left(\psi_{1}, \psi_{2}, \psi_{3}, \bar{\psi}_{4}, \bar{\psi}_{5}, \bar{\psi}_{6}\right)=\frac{\langle 12\rangle[56]\langle 3|1,2| 4] s_{12} s_{56}}{s_{124}} \\
& + \text { perms., } \\
& A_{4}^{\mathrm{DBI}}\left(\phi_{1}, \phi_{2}, \phi_{3}, \phi_{4}\right)=s_{12}^{2}+s_{23}^{2}+s_{13}^{2}, \\
& A_{6}^{\mathrm{DBI}}\left(\phi_{1}, \phi_{2}, \phi_{3}, \phi_{4}, \phi_{5}, \phi_{6}\right) \\
& =-s_{12} s_{34} s_{56}+\left(s_{12}^{2}+s_{23}^{2}+s_{13}^{2}\right)\left(s_{45}^{2}+s_{56}^{2}+s_{46}^{2}\right) \frac{1}{s_{123}} \\
& + \text { perms. }
\end{aligned}
$$

The general dimension BI amplitudes can be laborious to write out even at four points, but they can be given by

$$
\begin{aligned}
A_{4}^{\mathrm{BI}}=s t A_{4}^{\mathrm{YM}}= & {\left[4 \operatorname{Tr}\left(F_{1} F_{2} F_{3} F_{4}\right)-\operatorname{Tr}\left(F_{1} F_{2}\right) \operatorname{Tr}\left(F_{3} F_{4}\right)\right.} \\
& +\operatorname{cyclic}(1,2,3)] .
\end{aligned}
$$

The traces are over Lorentz indices of linearized momentum-space field strengths:

$$
F_{i}^{\mu \nu} \equiv p_{i}^{\mu} \epsilon_{i}^{\nu}-\epsilon_{i}^{\mu} p_{i}^{\nu}
$$

More generally, the full supersymmmetric DBI-VA amplitudes can be obtained via the double-copy procedure as

$$
A^{\mathrm{DBIVA}}=A^{\mathrm{SYM}} \otimes A^{\mathrm{NLSM}},
$$

more precisely given in terms of partial amplitudes by Eq. (110) or in terms of color-dual dressed cubic graphs as per Eq. (23).

\section{E. Gal and sGal}

The Galileon is a theory of scalars which originally appeared in the context of gravity models [84-86], also discussed in [87]. It is given by a Lagrangian of the form

$$
\mathcal{L}_{\mathrm{Gal}}=-\frac{1}{2}(\partial \phi)^{2}+(\partial \phi)^{2} \sum_{n=4}^{\infty} c_{n} \operatorname{det}_{n},
$$

where $\operatorname{det}_{n}=n ! \partial^{\left[\mu_{1}\right.} \partial_{\mu_{1}} \phi \ldots \partial^{\left.\mu_{n}\right]} \partial_{\mu_{n}} \phi$. The contact terms, or Galileon vertices, are neatly given by

$$
V_{n}=\operatorname{Det}\left(M^{a}\right),
$$

where $M^{a}$ is the $(n-1) \times(n-1)$ matrix obtained by removing any row $a$ and column $a$ from the matrix $M_{i j}=$ $p_{i} \cdot p_{j}, i, j=\overline{1, n}$. Although not obvious, permutation invariance follows from momentum conservation. Its scattering amplitudes are given by

$$
\begin{gathered}
A_{4}^{\mathrm{Gal}}=c_{4} V_{4}, \\
A_{5}^{\mathrm{Gal}}=c_{5} V_{5}, \\
A_{6}^{\mathrm{Gal}}=\left(c_{4}\right)^{2}\left(\frac{V_{4}(1,2,3, p) \times V_{4}(-p, 4,5,6)}{s_{123}}+\text { perms. }\right) \\
+c_{6} V_{6} .
\end{gathered}
$$

Finally, the special Galileon is a particular linear combination of the Galileon operators, which satisfies an even stronger Galileon symmetry [88,89], as well as a stronger $\mathcal{O}\left(\tau^{3}\right)$ Adler zero condition. Unlike the general Galileon, the special Galileon amplitudes can also be obtained via the adjoint double-copy procedure as

$$
A^{\mathrm{SGal}}=A^{\mathrm{NLSM}} \otimes A^{\mathrm{NLSM}} .
$$

\section{AMPLITUDE CONSTRAINTS}

\section{A. Locality}

Locality fixes ${ }^{1}$ the pole structure of the functions considered, and will be assumed throughout the paper. A local ansatz may be written as

$$
B_{n}^{\text {local }}=\sum_{i} \frac{N_{i}}{D_{i}}
$$

where, depending on the theory considered, the sum runs over all over cubic or quartic tree diagram topologies $i$ with corresponding massless scalar propagators $D_{i}$. The $N_{i}$ are polynomials of momenta (and polarization vectors for $\mathrm{BI}$ ), with unfixed coefficients, with their mass dimension fixed in terms of the net mass dimension of the amplitude. For NLSM, BI, and Gal, the quartic structure implies that each diagram in the ansatz will have exactly $n / 2-2$ poles, thus fixing the mass dimension of the numerators to $[n-2]$, $[2 n-4]$ and $[3 n-6]$ respectively. Terms with fewer (or zero) poles, such as contact terms, are (nonuniquely) included in the numerators. Since at this stage we are not yet assuming unitarity, these $N$ do not have any initial factorization properties.

We will assume for full generality and to maximize potential independence of kinematic invariants, that unless otherwise specified the spacetime dimension can be taken arbitrarily large, at least $D_{\mathrm{ST}}>n$ for any $n$-point amplitude in consideration.

\footnotetext{
${ }^{1} \mathrm{We}$ note that there is a certain ambiguity, present in the literature, regarding whether it is locality or unitarity that requires simple poles. Here we will use the term locality to denote a constraint on the properties of denominators, i.e., the presence of only simple poles, and the term unitarity to constrain the factorization properties of amplitudes on these simple poles.
} 


\section{B. Unitarity}

Unitarity further imposes that, on each pole $P^{2}, B_{n}^{\text {local }}$ factorizes into two lower point amplitudes:

$$
\lim _{P^{2} \rightarrow 0} B_{n}=\frac{A_{L} \times A_{R}}{P^{2}} .
$$

This implies that with unitarity the only unfixed piece of the ansatz is a potential contact term:

$$
B_{n}^{\text {unitary }}=[\text { factorizing piece }]+C_{n}\left(p^{m}\right),
$$

where the first part is fully determined by Eq. (48), and $C_{n}$ is now a polynomial of mass dimension $[m]$, with unfixed coefficients.

We find demanding unitarity an extremely sharp constraint that will allow us to prove many uniqueness claims. In most cases we will find additional evidence that unitarity surprisingly emerges as a consequence of locality and other properties.

\section{Soft limits}

Soft limits will be central to our arguments for uniqueness. Given their universality and usefulness, soft theorems have been under intensive recent study (see e.g., [14, 90-102] and references therein). We note however that we will not be assuming or imposing any of the soft theorems, but only using soft limits as formal Taylor expansions. In some cases, the soft theorems will in fact arise from UV constraints.

There are two closely related types of soft limits that we will use. First is the Adler zero, which involves taking one particle soft by rescaling one momenta $p \rightarrow \hat{p}=\tau p$, and taking $\tau \rightarrow 0$. In this limit, several special EFT's scale as

$$
A \rightarrow \mathcal{O}\left(\tau^{\sigma}\right),
$$

where $\sigma=1$ NLSM, $\sigma=2$ for DBI and Galileon, and $\sigma=3$ for sGal [9]. These particular values for $\sigma$ are interesting because they are below what simple mass dimension counting would imply. Take for instance the NLSM at six points:

$$
A_{6}=\frac{s_{13} s_{46}}{s_{123}}-s_{46}+(\text { cyclic }) .
$$

While each term separately scales as $\mathcal{O}\left(\tau^{0}\right)$ under a $p_{2} \rightarrow 0$ limit, their sum has an improved $\mathcal{O}\left(\tau^{1}\right)$ scaling. Such cancellations become highly nontrivial at higher points and for other theories like DBI or sGAL, and are in fact so powerful they fully constrain the theories $[1,2,8]$.

The other type of soft behavior relevant for EFT's is the double soft expansion [7,103]:

$$
A_{n+2} \rightarrow \tau^{\sigma}\left(S_{0}+\tau S_{1}+\ldots\right) A_{n} .
$$

In this case the nontrivial aspect is the factorization between the "soft factors" $S_{i}$ and the lower point amplitude $A_{n}$. Like the Adler zero, this expression places very stringent constraints on the amplitudes, in fact again sufficiently strong to fully constrain them [14]. For EFT's, this later claim relies on the following fact: there are no objects with enhanced double soft limits, except for the Galileon vertices.

These "uniqueness" results can be turned into very powerful tools, as they imply amplitudes are fully determined by just the first few orders in a soft expansion. Not only does this greatly simplify checks, in many cases it facilitates proofs through inductive arguments. Since we will use these results throughout the article, we can rephrase them more succinctly and practically:

(i) There are only four local objects which have an enhanced single soft limit: NLSM, DBI, Galileon vertex, sGal.

(ii) There is a unique local object which has an enhanced double soft limit: the Galileon vertex.

(iii) Anything else has a scaling dictated purely by mass dimension and singularity structure.

We should mention that these facts have not been proven rigorously for the Galileon or the special Galileon, but such proofs likely follow from arguments of the type given in [2]. For completeness, we will prove one particular case which shows up when discussing BI:

There is no polynomial of mass dimension $[n]$ with double soft scaling $\mathcal{O}\left(\tau^{3}\right)$.

\section{2S: Two-particle-shift scaling}

The BCFW shift [15] was originally introduced in four dimensions to enable a powerful on-shell recursion. Briefly, the recursion relies on using Cauchy's residue theorem to rebuild amplitudes from lower point information via unitarity. In $D$ dimensions, this is achieved via a scalar shift:

$$
\begin{aligned}
& p_{i} \rightarrow p_{i}+z q, \\
& p_{j} \rightarrow p_{j}-z q,
\end{aligned}
$$

subjected to $p_{i} \cdot q=p_{j} \cdot q=q^{2}=0$, needed to preserve the on-shell conditions, or a vector shift [26]:

$$
\begin{aligned}
\epsilon_{i} & \rightarrow \hat{\epsilon}_{i}, \\
\epsilon_{j} & \rightarrow \hat{\epsilon}_{j}+z p_{i} \frac{\hat{\epsilon}_{i} \cdot \epsilon_{j}}{p_{i} \cdot p_{j}}, \\
p_{i} & \rightarrow p_{i}+z \hat{\epsilon}_{i}, \\
p_{j} & \rightarrow p_{j}-z \hat{\epsilon}_{i},
\end{aligned}
$$

where $\hat{\epsilon}_{i}=\epsilon_{i}-p_{i} \frac{\epsilon_{i} \cdot p_{j}}{p_{i} \cdot p_{j}}$. In both cases we will refer to shifts as $[i, j\rangle$. 
If the amplitude vanishes for large $z$, it can be rebuilt purely from its residues in an extremely efficient manner:

$$
A_{n}=\sum_{k} \frac{A_{L}\left(z_{k}\right) A_{R}\left(z_{k}\right)}{P^{2}}
$$

where the sum runs over all channels where $P^{2}\left(z_{k}\right)=0$. Even if the amplitude does not vanish at large $z$, the recursion may be generalized to multiline shifts, and complemented by other properties, like the Adler zero for EFT's [9,18,21,23,24]; see also [67,104] for biadjoint scalar amplitudes. In any case, the scaling is crucial but difficult to compute. This is because very complicated cancellations occur such that the actual scaling is well below the naive expectation from power counting. The fact that these cancellations occur at all seems almost miraculous, and is a fact completely hidden from the Lagrangian perspective. The most well-known scalings are for YM and GR, which behave as $[25,105,106]$

$$
\begin{gathered}
A^{\mathrm{YM}} \sim \mathcal{O}\left(z^{-1}\right), \quad \text { for adjacent } i, j \\
A^{\mathrm{YM}} \sim \mathcal{O}\left(z^{-2}\right), \quad \text { for nonadjacent } i, j \\
A^{\mathrm{GR}} \sim \mathcal{O}\left(z^{-2}\right),
\end{gathered}
$$

making them perfect candidates for the BCFW recursion. In this article we find that EFT's also have an enhanced scaling at large $z$ :

$$
\begin{gathered}
A^{\mathrm{NLSM}} \sim \mathcal{O}\left(z^{1}\right), \quad \text { for adjacent } i, j \\
A^{\mathrm{NLSM}} \sim \mathcal{O}\left(z^{0}\right), \quad \text { for nonadjacent } i, j \\
A^{\mathrm{BI}} \sim \mathcal{O}\left(z^{0}\right), \\
\left\{A^{\mathrm{DBI}}, A^{\mathrm{Gal}}, A^{\mathrm{sGal}}\right\} \sim \mathcal{O}\left(z^{2}\right) .
\end{gathered}
$$

We have checked each of these scalings explicitly through various low multiplicities. It turns out that because of their double-copy structure, to know their $2 \mathrm{~S}$ scaling at all multiplicity, one only needs to know the scaling of their building blocks. Namely, knowing the scaling of NLSM and YM amplitudes is sufficient for all of the above amplitudes. All multiplicity Yang-Mills scaling are constrained by generalizations of action arguments found in Ref. [25], and while we expect that a similar all-orders argument exists for the NLSM, we do not pursue it here. Instead we simply verified NLSM BCFW scaling explicitly through 10 points.

The point of this article is however not to apply the recursion and construct amplitudes directly via unitarity, but instead show that particular amplitudes can be completely defined by demanding enhanced UV behavior, and that unitarity follows as a consequence. This result is particularly surprising for EFT's, for the following reason. In gauge and gravity theories, we have already seen that gauge invariance completely fixes the form of the amplitudes (both with unitarity [107] and without [1,2]). Given that the vector shift (55) seems to incorporate a gauge transformation, it is not difficult to believe the enhanced scaling implies gauge invariance, and hence fixes the amplitudes. For the EFT's under consideration however, it is the Adler zero that fixes contact terms. Yet how the Adler zero might be encoded in the shift (53) is even more mysterious. Not to mention that BI (as the off-spring of YM and NLSM - prime representatives of gauge invariance and Adler zero, respectively) is not fixed even by both, at least not directly. Via dimensional reduction, it was argued in [83] that BI may be fixed by the combination of Adler zero and gauge invariance. That the constraints following from such a complicated procedure can simply be turned into UV conditions is nevertheless quite surprising.

\section{E. SHS: Single-hard scaling}

The existence of on-shell recursion [15,108] for special constructible theories suggests there is value in considering the constraining information via on-shell quantities such as the BCFW shift we consider above. Given the utility of venerable Berends-Giele off-shell recursion approaches and related perturbiner methods (e.g., Refs. [109-112]) for scattering, it is perhaps a natural question to ask whether an off-shell constraint may provide sufficient information for a bootstrap constructibility program. We now introduce one such off-shell constraint, in the form of a single offshell "hard" limit. We will see that when combined with unitarity/factorization, at a specified mass dimension, the described single hard scaling can completely constrain the predictions of many theories. More tantalizing through explicit calculation in these theories at various accessible multiplicities, we find evidence to support conjectures that the single hard scaling alone can be sufficient to entirely constrain these amplitudes, in some sense allowing unitarity to emerge from such considerations.

Let us consider an amplitude: $A(\ldots p \ldots)$ where we want to take a single leg hard via $\hat{A_{n}}(\ldots \hat{p} \ldots)$ via a rescaling $p \rightarrow \hat{p}=z p$. We have to be careful because the momentum conserving delta function is in a sense trivialized in this limit

$$
\delta\left(z p+\sum_{i}^{(n-1)} p_{i}\right) \rightarrow \delta(z p),
$$

and the remaining momenta are poorly constrained, in contrast to the case of taking $z p$ soft, when momentum conservation can be dealt with consistently [113]. As one can use conservation of momenta to obscure the scaling of $p$, to unambiguously define a scaling, in a similar manner as when defining soft limits, we insist on using a $p$-favoring 
basis of momentum invariants that makes the $p$ dependence of $A_{n}$ explicit. Doing so requires only specifying a leg $i$ to always eliminate in favor of $p$, as well as a distinct momentum invariant $p_{j} \cdot p_{k}$ where $p_{j} \neq p_{k} \neq p_{i} \neq p$ to also be eliminated from the basis of invariants. As such one can label any a set of basis of momentum invariants that satisfy conservation of momentum and maximally favor the appearance of $p$ by a triplet: $\delta(i,[j, k])$ with the following defining properties:

(i) $\delta(i,[j, k])$ is any basis of momentum invariants that explicitly removes any reference to $p_{i}$ and $p_{j} \cdot p_{k}$ in its basis elements.

(ii) This can be accomplished by solving the set of equations generated by considering both $p_{m}^{2}=0$ and $\left(\sum_{l=1}^{n} p_{m} \cdot p_{l}\right)=0$ for every $m$, eliminating $p_{j} \cdot p_{k}$ and all $p_{i} \cdot p_{m}$ in favor of other momentum invariants.

(iii) Furthermore in the case of vector theories, enforcing $0=p_{m} \cdot \epsilon_{m}$ for all $m$, and eliminating either $\epsilon_{i} \cdot p_{j}$ or $\epsilon_{i} \cdot p_{k}$ in favor of $\epsilon_{i} \cdot p$ via $0=\sum_{m=1}^{n} p_{m} \cdot \epsilon_{i}$.

Once cast into an appropriate $p$-favoring basis of momentum invariants by applying $\delta(i,[j, k])$, the scaling of the hard limit can unambiguously be extracted, but will depend on the relative positions of the hard particle and the three particles singled out by $\delta(i,[j, k])$. The hard particle $p$ and particle $p_{i}$ separate the ordered set $\sigma=\{1, \ldots, n\}$ into two parts, $L$ and $R$ (either possibly empty):

$$
A\left(p, L, p_{i}, R\right) .
$$

Now, with respect to the separation $\sigma=\left(p, L, p_{i}, R\right)$, we define the set $\delta=(i,[j, k])$ as being

(1) compatible with $\sigma$, for $i$ not adjacent to $p$, and $\{j, k\} \in L$ or $\{j, k\} \in R$,

(2) not compatible, otherwise.

As a road map to the results presented in detail in Sec. V, we will summarize here what we discover about the singleshift UV behavior of the theories under consideration. Through explicit calculation in accessible multiplicities, YM and NLSM present the following enhanced behavior for ordered amplitudes when taking $z p$ to be large:

$$
\begin{gathered}
A_{n}(\sigma) \sim \mathcal{O}\left(z^{0}\right), \quad \text { for compatible ordering } \\
A_{n}(\sigma) \sim \mathcal{O}\left(z^{1}\right), \quad \text { otherwise. }
\end{gathered}
$$

The notation comes in handy for the biadjoint scalar, whose amplitudes are now

$$
A\left(\sigma_{1}, \sigma_{2}\right)=A\left(p, A_{1}, p_{i}, B_{1} \mid p, A_{2}, p_{i}, B_{2}\right) .
$$

With this notation, the biadjoint scalar scales as
$A_{n}\left(\sigma_{1} \mid \sigma_{2}\right) \sim \mathcal{O}\left(z^{-3}\right)$, for $\delta$ compatible with both $\sigma_{1}$ and $\sigma_{2}$

$$
\begin{aligned}
A_{n}\left(\sigma_{1} \mid \sigma_{2}\right) \sim \mathcal{O}\left(z^{-2}\right)(\text { or better }), \\
\text { for } \delta \text { compatible with either } \sigma_{1} \text { or } \sigma_{2} \\
A_{n}\left(\sigma_{1} \mid \sigma_{2}\right) \sim \mathcal{O}\left(z^{-1}\right)(\text { or better }), \quad \text { otherwise. }
\end{aligned}
$$

The biordered scaling is not completely determined because we have not taken into account the relative ordering between $\sigma_{1}$ and $\sigma_{2}$. Amplitudes with more "orthogonal" relative orderings can contain very few terms, and in this case even bad choices for $\delta$ can have improved scaling, which requires no cross-term cancellations. However, the above minimum requirements will be sufficient for our purposes. Next, for the full color-dressed NLSM and YM amplitudes, as well as GR, DBI, sGal and the Gal vertex we find:

$$
\begin{gathered}
\left\{\mathcal{A}^{\mathrm{YM}}, \mathcal{A}^{\mathrm{NLSM}}\right\} \sim \mathcal{O}\left(z^{1}\right), \\
\left\{A^{\mathrm{GR}}, A^{\mathrm{DBI}}, A^{\mathrm{sGal}}\right\} \sim \mathcal{O}\left(z^{3}\right), \\
V^{\mathrm{Gal}} \sim \mathcal{O}\left(z^{4}\right),
\end{gathered}
$$

independent of the choice for $\delta(i,[j, k])$ due to permutation invariance. Although surprising, this independence will be easily understood through the double-copy procedure. Furthermore, higher derivative corrections to any of these theories follow a similar pattern. Assuming $\kappa$ extra derivatives to NLSM, YM, GR, sGal, etc., we universally find

$$
A_{n}^{\kappa} \sim z^{\kappa / 2} A_{n}^{(\kappa=0)} .
$$

It should be mentioned that, like for the two-particle shift, intricate and quite unexpected cancellations between different Feynman diagrams are required to enable these scalings.

We have explicitly checked that each of these scalings holds through various low multiplicities. Because of their double-copy structure, as with the BCFW scaling, to know their single hard scaling at all multiplicity, one only needs to know the scaling of their building blocks, namely, NLSM and YM. In the single hard scaling case we do not have all multiplicity Yang-Mills or NLSM single hard scaling arguments available, and so explicitly verified Yang-Mills through $n=7$ and NLSM through $n=10$. We believe it would be interesting to pursue all-multiplicity proofs of this novel single hard scaling. Here we occupy ourselves with a different question: what additional constraints, beyond a particular SHS scaling (e.g., locality, unitarity, mass dimension, etc.), are required to uniquely specify the amplitudes of a given theory? We address these questions on a case-by-case basis in Sec. V.

One peculiarity of this scaling is that for ordered amplitudes, the (non)adjacency of $(n+1)$ and $i$ matters, 
similar to how it does for a BCFW shift. Another obvious feature is that the single hard limit essentially looks like "half" of a BCFW shift. The exact difference is easy to quantify. The two-line particle shift (53) does not affect momentum conservation, so the overall scaling is independent of the triplet $\delta(i,[j, k])$. Choose $i=n$ and consider a $[1, n\rangle$ shift. Because $p_{n}$ does not appear explicitly, the shift actually reduces to a single deformation:

$$
p_{1} \rightarrow p_{1}+z q,
$$

subjected to $q \cdot p_{1}=0$ and $q \cdot\left(\sum_{i=1}^{n-1} p_{i}\right)=0$. It is now clear that to obtain the single hard limit we need only set $q=p_{1}$ and drop the on-shell condition $q \cdot\left(\sum_{i=1}^{n-1} p_{i}\right)=0$. Conversely, a full momentum-conserving two-particle shift can simply be obtained from a single hard limit by imposing the extra on-shell condition $q \cdot\left(\sum_{i=1}^{n-1} p_{i}\right)=0$. An immediate consequence of this fact is that the behavior under a two-particle shift cannot be worse than under a single hard limit.

While apparently ill defined because of momentum conservation issues, unlike the typical high energy limits involved in the Froissart bound [114], the single hard limit is nevertheless a valid and natural kinematic configuration to explore. It turns out to be a nontrivial property of many theories, and in fact a defining property of NLSM and sGal. It would be interesting nonetheless if the enhanced behavior has any implications for the Froissart bound itself.

\section{AMPLITUDE RELATIONS AND THE NLSM}

The trace basis (27) is not minimal, and can be further reduced two times. First, the Kleiss-Kuijf (KK) amplitude relations [115]

$$
A_{n}(1, \alpha, n, \beta)=(-1)^{|\beta|} \sum_{\sigma \in \alpha|| \beta^{T}} A_{n}(1, \sigma, n),
$$

where $\alpha$ and $\beta$ are lists of external labels, $\beta^{T}$ represents the reverse ordering of the list $\beta$, and $\alpha \sqcup \beta^{T}$ are the permutations that shuffle the $\alpha$ and $\beta$, i.e., that separately maintain the relative order of the elements belonging to each list but can interleave elements from both lists. These relate the $(n-1)$ ! ordered amplitudes, $A(1, \tau)$, amplitudes to an $(n-2)$ ! basis with two legs fixed: $A(1, \sigma, n)$. Writing the adjoint generator matrices as $\left(f^{a}\right)_{b c} \equiv f^{b a c}$ one can write any flavor factor as products of $f^{a_{i}}$ 's. This leads to the following expression for the full flavor-dressed amplitude [72]:

$$
\mathcal{A}_{n}^{\text {tree }}=g^{n-2} \sum_{\sigma \in S_{n-2}} A_{n}^{\text {tree }}(1, \sigma, n) c(1|\sigma| n),
$$

where $c(1|\sigma| n)$ is the color-weight of the cubic half ladder graph with farthest legs 1 and $n$ fixed and the intermediate legs labeled according to $\sigma$. Second, because the NLSM in the adjoint obeys color kinematics [116], this implies a further reduction to a basis of $(n-3)$ ! independent amplitudes. This necessarily manifests in the satisfaction of the simplest, or so-called fundamental BCJ, relations [27,117] which can be written as

$$
\sum_{i=2}^{n-1} k_{1 i} A(2, \ldots, i, 1, i+1, \ldots, n)=0,
$$

where $k_{1 i}=\sum_{j=2}^{i} p_{1} \cdot p_{j}$.

A key consequence of the adjoint double-copy structure is that at tree-level color-dual kinematic graph weights can be given by specifying the kinematic numerators of $(n-2)$ ! half-ladder master graphs, deriving all other weights by Jacobi. One representation for Jacobi-satisfying kinematic weights for the nonlinear sigma model is simply

$$
n^{\mathrm{NLSM}}(1|\sigma| n)=S(\sigma \mid \sigma),
$$

where $S$ is the celebrated $(n-2)$ ! rank KLT matrix given below in Eq. (111). This form was conjectured in Ref. [79] and proven from string-theoretic considerations in [118]. This intimate relation between the KLT matrix and NLSM amplitude is a first hint of the deeper fact we prove here, that NLSM amplitudes are uniquely specified by the quartic structure and color kinematics.

The NLSM further plays a central role in the other theories we will consider:

(1) supersymmetric DBI-VA: NLSM $\otimes$ supersymmetric Yang-Mills, and

(2) special Galileon: NLSM $\otimes$ NLSM.

Each of these theories has diverging high-energy behavior and so can be understood as effective field theories requiring some sort of completion in the UV. The NLSM itself finds a UV completion in Abelian Z-theory [79] and the (supersymmetric) DBI-VA has a UV completion in the Abelian supersymmetric open string. While each of the double-copy factors above can admit higher-derivative corrections, it is interesting to note that both Z-theory and the Abelian open string only exploit higher-derivative corrections to their respective pion factors. Indeed both UV completions receive higher derivative corrections to their pion factors in the same ratio, as the Abelian open string at tree level can be understood as a field theory double copy between Abelian Z-theory amplitudes and supersymmetricYang-Mills. As we will see, the combination of locality, higher derivatives, and amplitude relations in combination with unitarity can be highly constraining.

\section{A. Uniqueness from amplitude relations}

Here we finally explore the space of local objects that can obey the color-kinematic duality, or equivalently, the amplitude relations. Imposing the amplitude relations 
rather than graph-level numerator constraints in this case is much more efficient. To compare, an ordered quartic amplitude scalar ansatz at eight points has 18,540 terms, which are subject to just two constraints: cyclic invariance and the BCJ relations. On the other hand, a general cubic ansatz for the eight-point half-ladder graph relevant to NLSM power counting has 177,100 terms, which must satisfy antisymmetry about each vertex and the Jacobi relations about each propagator, as well as vanishing of all cubic residues of the resulting ordered amplitudes.

Claim 1 Flavor-ordered (pionic) NLSM amplitudes are fixed uniquely by locality, [mass dimension] $=2$, cyclic invariance, and the $\mathrm{BCJ}$ relation:

$$
F_{n}=\sum_{i=2}^{n-1} k_{1 i} A_{i ; n}(2, \ldots, i, 1, \ldots, n)=0,
$$

where $k_{1 m}=\sum_{i=2}^{m} p_{1} \cdot p_{i}$.

We do not need to assume that the $A_{i ; n}$ are related to each other by relabeling, so this is in fact a stronger statement than the numerator level duality. To prove this claim we will make use of the Adler zero uniqueness. Taking the first particle soft as $p_{1}=\tau p_{1}$, with $\tau \rightarrow 0$, we require that $F_{n}$ vanishes order by order in $\tau$ :

$$
F_{n}(\tau) \rightarrow \tau F^{(1)}+\tau^{2} F^{(2)}+\ldots=0,
$$

which will involve the soft limit expansion of the amplitudes:

$$
A_{n}(\tau) \rightarrow \tau^{0} A_{n}^{(0)}+\tau A_{n}^{(1)}+\ldots
$$

Because of the quartic propagator structure, no pole in $A(n)$ is singular in this limit, hence at leading order the dependence on the soft momenta drops out:

$$
\begin{aligned}
A_{n}(\tau) & =\sum \frac{N\left(\tau p_{1}\right)}{D\left(\tau p_{1}\right)} \\
& =\sum \frac{N^{(0)}+\tau p_{1} N^{(1)}+\ldots}{D^{(0)}+\tau P^{(1)}+\ldots} \rightarrow \frac{N(0)}{D(0)}+\ldots \\
& =A_{n}^{(0)}+\ldots
\end{aligned}
$$

Now we impose

$$
F^{(1)}=\sum_{i} k_{1 i} A_{n ; i}^{(0)}(1, \ldots, i, \ldots, n)=0 .
$$

The $k_{1 i}$ coefficients in front of each $A_{i}$ are independent (under $n$-point kinematics) and since the $A_{i}$ 's themselves are independent of $p_{1}$, the above equation implies that each $A_{n}^{(0)}$ must vanish separately. But this is precisely the Adler zero condition for particle 1 . Using cyclic invariance it means that each $A_{i}$ must satisfy the Adler zero in all $n$ particles, and there is a unique local object with this property: the NLSM amplitude.

\section{B. Higher-derivative corrections from amplitude relations and unitarity}

The amplitude relations (80) also put stringent constraints on higher derivative corrections to the NLSM [24]. While not sufficient just with locality, we find that also assuming unitarity can uniquely determine such amplitudes, to some finite mass dimension. To test this claim we can write an ansatz:

$$
B_{n}^{\mathrm{NLSM}+\mathrm{H} . \mathrm{D} .}=[\text { factorizing piece }]+C_{n}\left(p^{\kappa}\right),
$$

where the factorizing part is fully determined by unitarity, while the contact term $C_{n}$ is a polynomial. If we consider theories coming from $\mathcal{O}\left(p^{\kappa}\right)$ operators, then this polynomial has mass dimension [ $[$ ], with $\kappa=2$ corresponding to the usual NLSM amplitude. We have found that at six points, up to $\kappa=10$, the contact term cannot satisfy the BCJ relations on its own, and therefore unitarity plus amplitude relations fix the ansatz uniquely. For $\kappa=12$ there are two polynomial solutions to the $\mathrm{BCJ}$ relations.

One has to go to $\kappa=14$ at four points to find the first analogous situation where two independent colorkinematic satisfying solutions occur, but it is instructive to explore. Quite simply. the $s, t$ channel has the following two independent solutions:

$$
A^{[14]}(s, t)=u\left(\alpha(s t u)^{2}+\beta\left(s^{6}+t^{6}+u^{6}\right)\right) .
$$

After modding out the $u$ required to satisfy the four-point $(n-3)$ ! relations we are left with two independent permutation invariant basis elements of the correct dimension. Both contribute to Abelian Z-theory (and consequently the Abelian open string) at the $\alpha^{\prime 8}$ order, suggesting that their coefficients may be ultimately fixed by massive mode resonance unitarity considerations of the UV completion (cf. e.g., Ref. [119]). It would be fascinating if other conditions can be imposed to uniquely fix even these higher $\kappa$ ansätze, but also to understand the structure of these special polynomials.

\section{UNIQUENESS FROM UV BEHAVIOR}

\section{A. Doubly ordered biadjoint amplitudes}

Claim 2 The doubly ordered biadjoint amplitudes are fixed uniquely by locality and the following UV single hard scaling $z p_{n} \rightarrow \infty, \delta(i,[j, k])$ :

$$
\begin{aligned}
& A_{n}\left(\sigma_{1} \mid \sigma_{2}\right) \sim \mathcal{O}\left(z^{-3}\right), \\
& \text { for } \delta \text { compatible with both } \sigma_{1} \text { and } \sigma_{2}, \\
& A_{n}\left(\sigma_{1} \mid \sigma_{2}\right) \sim \mathcal{O}\left(z^{-2}\right),
\end{aligned}
$$$$
\text { for } \delta \text { compatible with either } \sigma_{1} \text { or } \sigma_{2} \text {. }
$$ 
As mentioned before, the amplitudes can have even better scalings in particular situations. However, the above conditions are sufficient to fully determine all biadjoint amplitudes. The proof is a much simpler version of the argument applied to Yang-Mills in Ref. [26]. Briefly, we only need to show that the leading soft piece of the biadjoint amplitude is fixed uniquely by imposing UV constraints. This is because any term in $\phi^{3}$ amplitudes, for four point and above, has at least two cubic poles, and therefore shows up in at least one single soft theorem. Hence the biadjoint is completely fixed by just its leading soft theorem, similar to other cases as explained in Ref. [14]. We carry out the proof by induction in Appendix A, for the simplifying case when $\sigma_{1}=\sigma_{2}=(1,2, \ldots, n)$, as other configurations follow from identical reasoning.

Here we examine instead the first step of the induction, that UV constraints fix the five-point amplitude. The check is simple, but it is instructive, as unlike the examples to follow, the biadjoint has trivial numerator structure, and obeys no on-shell constraints other than unitarity (with simple soft theorems as a consequence) and amplitude relations once it is dressed with a single copy of color factors. It therefore most transparently demonstrates that improved UV scaling is directly tied to unitarity. Consider the five-point example for $\sigma_{1}=\sigma_{2}=(1,2,3,4,5)$, and take $z p_{1}$ large, choosing $\delta(3,[4,5])$

$$
\begin{aligned}
A_{5}= & \frac{a_{1}}{s_{12} s_{34}}+\frac{a_{2}}{s_{23} s_{45}}+\frac{a_{3}}{s_{34} s_{51}}+\frac{a_{4}}{s_{45} s_{12}}+\frac{a_{5}}{s_{51} s_{23}} \\
\rightarrow & \frac{1}{z^{2}}\left(\frac{a_{1}}{p_{1} \cdot p_{2}\left(p_{1} \cdot p_{2}+p_{1} \cdot p_{5}\right)}+\frac{a_{3}}{p_{1} \cdot p_{5}\left(p_{1} \cdot p_{2}+p_{1} \cdot p_{5}\right)}+\frac{a_{4}}{p_{1} \cdot p_{2}\left(p_{1} \cdot p_{2}+p_{1} \cdot p_{4}+p_{1} \cdot p_{5}\right)}\right. \\
& \left.-\frac{a_{5}}{p_{1} \cdot p_{2}\left(p_{1} \cdot p_{2}+p_{1} \cdot p_{4}+p_{1} \cdot p_{5}\right)}-\frac{a_{5}}{p_{1} \cdot p_{2} p_{1} \cdot p_{5}}\right)+\mathcal{O}\left(z^{-3}\right) \\
= & \mathcal{O}\left(z^{-3}\right) .
\end{aligned}
$$

All five terms contribute at order $\mathcal{O}\left(z^{-2}\right)$ and are needed for the enhancement, meaning that the SHS is probing several cuts at the same time, while any regular unitarity constraint could only probe at most two diagrams at a time. We obtain two constraints: $a_{1}+a_{2}-a_{5}=0$ and $a_{3}-a_{4}=0$, with the remaining two obtained by other hard limits, leading to $a_{1}=a_{2}=a_{3}=a_{4}=a_{5}$, fixing the five-point amplitude.

The above example also shows another quite amusing property of the SHS: it can tell that quantities which apparently look like singly ordered $\phi^{3}$ partial amplitudes are properly resolved by definition as doubly ordered biadjoint amplitudes. Consider the simple example of Eq. (89), which reads like an ordered cubic scalar amplitude. If we make a "bad" choice for $\delta(i,[j, k])$, we discover that this object scales as $\mathcal{O}\left(z^{-1}\right)$, two powers of $z$ worse than (91). Comparing with all the other known singly ordered partial amplitudes, for which the difference between "bad" and "good" $\delta(i,[j, k])$ is just one power of $z$, this seems like a puzzle. The discrepancy is easily explained by the rules given by (69), which seem to suggest we are in fact making a "doubly" bad choice. If we are to trust these rules as fundamental, the resolution is obvious: such a scalar amplitude carries an extra hidden identical ordering, so our choice for $\delta(i,[j, k])$ is breaking the rule twice.

Next we move on to more complicated theories, which have extra kinematic structure in the numerators. If the biadjoint scalar example seems to imply the UV scalings are perhaps just compactly imposing unitarity, we will see much more is true. As discussed previously, general theories (and in particular EFT's) have contact terms, which are invisible to factorization constraints. Therefore, somehow, UV scalings are imposing other symmetries on the amplitudes, and not just factorization.

\section{B. NLSM}

Claim 3 NLSM amplitudes are fixed uniquely by locality, unitarity [mass dimension] $=2$, and the following UV behaviors:

(1) Single hard scaling $z p_{n} \rightarrow \infty$, with $\delta(i,[j, k])$ :

$$
\begin{gathered}
A_{n}(\sigma) \sim \mathcal{O}\left(z^{0}\right), \quad \text { for } \delta \text { compatible with } \sigma, \\
A_{n}(\sigma) \sim \mathcal{O}\left(z^{1}\right), \quad \text { otherwise. }
\end{gathered}
$$

(2) Two particle shift $[i, j\rangle(53)$ :

$$
\begin{aligned}
A_{n} & \sim \mathcal{O}\left(z^{1}\right), \quad \text { for adjacent } i, j, \\
A_{n} & \sim \mathcal{O}\left(z^{0}\right), \quad \text { for nonadjacent } i, j .
\end{aligned}
$$

We only need to show that any contact terms cannot independently satisfy the required scaling. Since it is a simple linear combination of kinematic invariants, the proof 
is straightforward via soft limits and induction. Using $p_{n+1}=\tau p_{n+1}$, we expand the contact term at $(n+1)$ around $\tau=0$ :

$$
C_{n+1}=\tau^{0} C_{n+1}^{(0)}+\mathcal{O}(\tau) .
$$

The leading piece $C_{n+1}^{(0)}$ is simply the lower point ansatz $C_{n}$, which by assumption cannot satisfy the SHS or $2 \mathrm{~S}$ constraints. Therefore $C_{n+1}$ scales as $\mathcal{O}(\tau)$ in the single soft limit, and is therefore ruled out by uniqueness from the Adler zero.

We expect that in fact the UV constraint is much more powerful, and that unitarity follows as a consequence. To test this claim, we setup a local ansatz over quartic graphs of the form:

$$
B_{n}^{\mathrm{NLSM}}=\sum_{i} \frac{N_{i}\left(p^{n-2}\right)}{D_{i}} .
$$

Possible contact terms are included in the numerators. Imposing either of these enhanced scalings, we have verified analytically that up through eight points the ansatz is completely fixed. The check can be performed easily at four and six points, where the ansätze have just 2 and 135 terms respectively, but grow relatively quickly: already at eight points the relevant ansatz has 18,480 terms.

\section{C. (Special) Galileon}

As a prelude to the special Galileon, we make two observations about the Galileon vertex, and the (general) Galileon amplitude. Because it will be useful later, we mark the following claim:

Conjecture 1 The Galileon vertex is uniquely fixed by $[$ mass dimension] $=[2 n-2]$ and the following UV behaviors:

(1) Single hard scaling (SHS): $V_{n} \sim \mathcal{O}\left(z^{4}\right)$

(2) Two-particle shift (2S): $V_{n} \sim \mathcal{O}\left(z^{2}\right)$.

We have verified these claims up through $n=7$, using a polynomial ansatz.

Proving the Galileon vertex actually has these scalings is simple. Recalling the definition (42), we choose $a=n$, and apply a $[1, n\rangle$ shift. Since $p_{n}$ does not appear in the matrix, the only $z$ contributions can come from $p_{1}$ in row 1 and column 1. Therefore the maximum power of $z$ in the determinant is 2 . For the single limit, extra $z$ contributions can come in the entries of $M$ which are removed via the on-shell constraint, say $p_{2} \cdot p_{3}$. There are two such entries, providing therefore two extra powers of $z$, for a total maximum power of 4 for the SHS.

Going beyond just the contact terms, the full Galileon, given by the general Lagrangian (41), itself curiously follows from imposing both the SHS and $2 \mathrm{~S}$ scaling and locality, at least up to $n=7$. Unlike the previous cases, here the coefficient of the contact term is not fixed relative to the factorizing piece (since the contact term is a solution), but surprisingly the factorizing piece itself is fixed.

Claim 4 Assuming conjecture 1, the special Galileon amplitudes are uniquely fixed by locality, unitarity, $[$ mass dimension $]=[2 n-2]$, and the single hard limit scaling:

$$
A_{n}^{\mathrm{sGal}} \sim \mathcal{O}\left(z^{3}\right)
$$

As with previous unitarity proofs above, the claim is that the only contact terms which satisfy the scaling do so by cancellation against factorization channels so can be completely fixed in that way. We proceed to rule out any additional contact terms that would satisfy the scaling in isolation. From the observation made in Sec. III E, an $\mathcal{O}\left(z^{3}\right)$ SHS scaling of a contact implies at least a $\mathcal{O}\left(z^{3}\right)$ scaling under the hard BCFW double shift (2S scaling). This $2 \mathrm{~S}$ scaling is automatically improved to $\mathcal{O}\left(z^{2}\right)$ by permutation invariance [120], which implies that the contact must uniquely be Galileon by conjecture 1 . But via the same conjecture, such a vertex has a $z^{4}$ SHS, so would violate the specified scaling condition of $z^{3}$.

We can see what happens if we do not impose unitarity. The ansatz in this case is

$$
B_{n}^{\mathrm{sGal}}=\sum_{i} \frac{N_{i}\left(p^{3 n-6}\right)}{D_{i}} .
$$

Because of the higher mass dimension of the numerators, only the $n=4$ and $n=6$ cases are straightforward to verify. We find in these cases that the ansatz is fixed, and unitarity emerges from the UV constraints.

The parallel to the IR story is interesting to note. There, the special Galileon was selected by demanding a further improved $\mathcal{O}\left(\tau^{3}\right)$ soft limit, up from the $\mathcal{O}\left(\tau^{2}\right)$ satisfied by the general Galileon. The corresponding scalings in the hard limit are on the other hand $\mathcal{O}\left(z^{4}\right)$ for the Galileon, improved to $\mathcal{O}\left(z^{3}\right)$ for the sGal.

\section{Born-Infeld}

Claim 5 BI amplitudes are uniquely fixed by locality, unitarity, $[$ mass dimension $]=n$, and a BCFW shift (55) scaling of

$$
A_{n}^{\mathrm{BI}} \sim \mathcal{O}\left(z^{0}\right)
$$

As before, we only need to show the contact term cannot independently scale, without engagement with terms on factorization channels, as $\mathcal{O}\left(z^{0}\right)$ under BCFW shifts. To do this, we expand in a double soft limit

$$
C_{n+2}^{\mathrm{BI}}=\tau^{0} C_{n+2}^{(0)}+\tau C_{n+2}^{(1)}+\ldots,
$$


and show that the BCFW constraint rules out $C^{(0)}, C^{(1)}$, and $C^{(2)}$. This is sufficient to rule out the whole term, since it is a polynomial of mass dimension $[n+2]$, and it cannot have a soft limit scaling of $\mathcal{O}\left(\tau^{3}\right)$ in all possible double soft limits, a proof we leave to the Appendix. In this setup the $[n+1, n+2\rangle$ shift is very constraining. At the first order, demanding $\mathcal{O}\left(z^{0}\right)$ under this shift fixes

$$
C_{n+2}^{(0)}=\epsilon_{n+1} \cdot \epsilon_{n+2} C_{n}\left(p^{n+2}\right),
$$

where $C_{n}\left(p^{n+2}\right)$ is some $n$-point general polynomial. Taking advantage of the fact that $p_{n}$ can be removed from $C_{n}$ via momentum conservation, we now impose an $[n+2, n\rangle$ shift. Since $C_{n}$ is linear in $e_{n}$, the shift produces a piece proportional to $z$, which cannot cancel against anything, and therefore $C_{n+2}^{(0)}$ must vanish. The next orders are slightly more involved but can be fixed using similar arguments, proving that BCFW scaling plus unitarity fixes the ansatz uniquely.

It is already quite surprising that BI can also be fixed simply by its high energy behavior, even without assuming gauge invariance or a Lagrangian form like (31), but we find evidence that even unitarity may be dropped from the starting assumptions. This is easily verified at four points, where the BI amplitude coincides with the numerator of the YM amplitude. However, even at six points the ansatz starts to become prohibitively large, containing over $3 \times 10^{6}$ terms. To check the conjecture we therefore Taylor expand the ansatz in a double soft limit, and check whether the ansatz is fixed order by order:

$$
B_{6} \rightarrow \frac{1}{\tau} B_{6}^{(-1)}+\tau^{0} B_{6}^{(0)}+\tau B_{6}^{(1)}+\tau^{2} B_{6}^{(2)}+\ldots
$$

We have indeed verified that imposing the scaling is enough to uniquely fix all terms up to and including $\tau^{2}$, and according to the arguments of the type given in Ref. [14], this is sufficient to fully fix the amplitude.

\section{Supersymmetric DBI-VA}

The UV constraints can be applied to 4D kinematics as well. We find that the photon and fermion sectors of DBIVA in four dimensions are uniquely fixed by locality, mass dimension, helicity weight, and two particle shift scalings:

$$
\begin{gathered}
A^{\text {photon }} \sim \mathcal{O}\left(z^{0}\right) \quad \text { for }(-,-),(+,+) \text { and }(-,+) \\
A^{\text {photon }} \sim \mathcal{O}\left(z^{4}\right) \quad \text { for }(+,-), \\
A^{\text {fermion }} \sim \mathcal{O}\left(z^{0}\right) \quad \text { for }(-,-) \text { and }(+,+) \\
A^{\text {fermion }} \sim \mathcal{O}\left(z^{1}\right) \quad \text { for }(-,+) \\
A^{\text {fermion }} \sim \mathcal{O}\left(z^{3}\right) \quad \text { for }(+,-) .
\end{gathered}
$$

For DBI-VA, we can write a local 4D ansatz:

$$
B_{n}=\sum \frac{N}{D}
$$

where the $N$ are polynomials of spinor dot products $\langle i, j\rangle$ and $[i, j]$ have mass dimension $[2 n-2]$ and a corresponding helicity weight for each particle. We have checked the conjecture up through $n=8$.

The scalar part of this theory, DBI, is a surprising exception in this context. Even though the infrared properties are enough to constrain it, the UV behavior apparently is not. Technically, this is because it is lower mass dimension than the Galileon, but obeys the same UV scalings $\left[\mathcal{O}\left(z^{2}\right)\right.$ BCFW shift, $\mathcal{O}\left(z^{3}\right)$ SHS].

\section{E. The double copy and UV behavior}

The double-copy procedure makes it clear how BI and sGal inherit the SHS UV scalings from YM and NLSM (see also Ref. [121,122] for discussions on the usual BCFW shift). We will use the following KLT representation:

$M_{n}=\sum_{\sigma(\alpha), \sigma(\beta)} A(1, \sigma(\alpha), n, n-1) S[\alpha \mid \beta] A(1, \sigma(\beta), n-1, n)$

where the KLT matrix can be defined recursively as

$S[A, j \mid B, j, C]_{i}=\left(k_{i B} \cdot k_{j}\right) S[A \mid B, C]_{i}, \quad S[\varnothing \mid \varnothing]_{i} \equiv 1$,

with $k_{i B} \equiv k_{i}+k_{b_{1}}+\cdots+k_{b_{|B|}}$, and we choose $i=1$.

This form is convenient because particles $n$ and $n-1$ are always adjacent on the right-hand side of Eq. (110), and furthermore $p_{n-1}$ and $p_{n}$ do not appear manifestly in the KLT matrix. Therefore, imposing the shift $[n-1, n\rangle$, we find the scalings

$$
\begin{gathered}
{[\mathrm{BI}]=[\mathrm{YM}]+[\mathrm{NLSM}]=(-1)+(1)=0,} \\
{[\mathrm{sGal}]=[\mathrm{NLSM}]+[\mathrm{NLSM}]=2 .}
\end{gathered}
$$

as expected. For the SHS, we can make the scaling manifest with any choice $\delta\left(p_{n-1},\left[p_{1} \cdot p_{i}\right]\right)$, taking $n$ hard. Since $n$ and $n-1$ do not appear explicitly, the only $p_{n}$ contribution can come from eliminating $p_{1} . p_{i}$. It is easy to see that any term of the type $p_{1} \cdot p_{i}$ appears exactly once in every $S[\alpha \mid \beta]_{1}$, contributing one power of $z$. We therefore obtain a SHS scaling

$$
[\mathrm{sGal}]=[\mathrm{NLSM}]+\left[S_{\mathrm{KLT}}\right]+[\mathrm{NLSM}]=3,
$$

as expected. The same argument shows $[\mathrm{GR}]=[\mathrm{BI}]=3$. 


\section{SUMMARY AND OUTLOOK}

To summarize, we have considered both color-kinematic and UV constraints on tree-level scattering amplitudes, novelly introducing a single-particle hard shift scaling. In conjunction with unitarity we proved a number of uniqueness claims, and collected evidence that for many of these theories unitarity could emerge from such constraints alone. As such results could potentially be far more reaching, suggesting structure that allows unitarity to follow from UV behavior, they are worth summarizing separately. The following quantities may be uniquely fixed by UV conditions:

(1) NLSM amplitudes (verified through eight points).

(2) The Galileon vertex (verified through seven points).

(3) The special Galileon amplitude (verified through six points).

(4) Arbitrary dimension Born-Infeld (verified through six points).

(5) 4D supersymmetric DBI-VA vector and fermion amplitudes (verified through eight points).

These results extend the list of cases when amplitudes can be derived solely from new principles. This makes it increasingly plausible that a different formulation exists, where some of these properties are primary, at the expense of manifest factorization and space-time descriptions. This is concordant with the "amplituhedron" program [123] (and recent generalizations [124]), where both locality and unitarity follow from more basic geometric principles.

Since these are all massless theories it is perhaps not surprising that the IR and UV limits actually contain equivalent information. However, a clear way to go from one to the other is still lacking. It would be interesting if the recently discovered conformal symmetry in $D$-dimensional YM and GR tree amplitudes plays any role in this context [125]. It is also likely that some symmetry must be behind these high energy limits for EFT's, similar to the "enhanced spin symmetry" that was discovered for YM and GR [25].

Since the spectrum of multiparticle theories, unless hardpartitioned into some sort of Grassmanian indexed generator (as is frequently done by defining an on-shell superspace) can often be inaccessible except at the (multi)loop level, it will be interesting to see what kind of constraints one can expect on gauge-invariant components of integrands. This is a program that has already produced interesting results considering generalized unitarity cuts [126]. Given the intimate relation between supersymmetry and UV behavior, it will not be surprising if UV scaling can reduce the number of cuts required to completely specify supersymmetric gauge theory integrands. A particularly interesting avenue would be to investigate any barriers to relating such UV scaling in nonsupersymmetric theories to their beta functions (cf. Ref. [127]).

We also note that in the context of celestial amplitudes (cf. Refs. [128-130]), the UV scaling of amplitudes makes an appearance, as does the infrared behavior (cf. Ref. [131-134]). The Mellin transform is an integral over the energies of the amplitude, and is sensitive to UV divergences, hence string theory completions are needed for consistency even when discussing purely gravitational amplitudes [135]. As the transform mixes the IR and UV of the amplitudes, any possible IR/UV connection may ultimately have an impact on celestial amplitudes as well.

\section{ACKNOWLEDGMENTS}

We thank Zvi Bern, Marco Chiodaroli, Henrik Johansson, Karol Kampf, Ian Low, Radu Roiban, Jaroslav Trnka, Ingrid Vazquez-Holm, Congkao Wen, Zhewei Yin, and Suna Zekioglu for many useful and interesting related discussions. J. J. M. C. and L. R. are supported by the European Research Council under ERC-STG-639729, Strategic Predictions for Quantum Field Theories.

\section{APPENDIX A: BIADJOINT SOFT THEOREM FROM UV SCALING}

We wish to prove that the leading order soft theorem of biadjoint scalar, given by

$$
A_{n+1}(1,2, \ldots, n) \rightarrow\left(\frac{1}{p_{n+1} \cdot p_{1}}+\frac{1}{p_{n+1} \cdot p_{n}}\right)
$$

can be fixed by the SHS scalings of claim 2. Since we are only dealing with the case $\sigma_{1}=\sigma_{2}$, the constraints simplify, and we can compactly refer to them as

$$
\begin{aligned}
E_{n} & \equiv \lim _{z p \rightarrow \infty} A_{n} \delta(i,[j, k]) \\
& \sim \mathcal{O}\left(z^{-3}\right), \quad \forall p, i \text { not adjacent to } p, \text { and } j>i \text {, or } k<i .
\end{aligned}
$$

As will become clear immediately, we in fact need to prove a somewhat stronger statement for the inductive argument to close: we impose the scalings for all particles, except one we denote $h$. Using our previous notation, further define

$$
E_{n}(h) \equiv E_{n}, \quad \forall p \neq h .
$$

Now we begin the inductive argument by assuming $A_{n}(\sigma)$ is fixed uniquely by the constraints $E_{n}(h), \forall h=\overline{1, n}$. We wish to show that $E_{n+1}\left(h^{\prime}\right)$ acting on a local ansatz $B_{n+1}$ implies $E_{n}(h)$ constraints acting on a lower point ansatz $B_{n}$, which is then fixed. Taking a soft limit,

$$
B_{n+1}(\sigma, n+1) \rightarrow \frac{B_{n ; 1}(\sigma)}{p_{n+1} \cdot p_{1}}+\frac{B_{n ; n}(\sigma)}{p_{n+1} \cdot p_{n}},
$$

where, because of the factorizing propagator structure, $B_{n ; i}$ are two different lower point local ansätze. Now the need to 
single out a particle $h$ becomes clear. If we were to take $p_{1}$ (or $p_{n}$ ) hard, demanding $\mathcal{O}\left(z^{-3}\right)$ on $B_{n+1}$, because of the denominators, this would only translate to a constraint $\mathcal{O}\left(z^{-2}\right)$ on $B_{n ; 1}$ (or $B_{n ; n)}$ ), and the inductive argument would not close. Even less subtly, once we have taken $n+1$ soft, we cannot of course take it hard, also leading to the induction not closing. We can resolve both these issues by starting from a slightly weaker constraint, where we do not impose the scaling for some particular particle $h$. We merely need to choose $h=n+1$ for the high point ansatz, and $h=1(h=n)$ for $B_{n ; 1}\left(B_{n ; n}\right)$, solving both problems at once.

It follows quickly that all constraints in $E_{n+1}(n+1)$, applied to (A4), translate to equivalent constraints $E_{n}(1)$ [or $E_{n}(n)$ ] applied to their respective $B_{n ; 1}$ (or $B_{n ; n}$ ), except two special choices: taking $p_{1}$ hard with $\delta(n, a, b)$ or taking $p_{n}$ hard with $\delta(1, a, b)$, for some $a$, $b$. For the moment, we just note that since 1 and $n$ are adjacent at $n$-points, and so are in fact not part of $E_{n}$ as defined by (A2). Therefore, by assumption we have enough constraints to fix the lower point ansätze, each up to some coefficient:

$$
B_{n+1} \rightarrow \frac{a_{1} A_{n}}{p_{n+1} \cdot p_{1}}+\frac{a_{n} A_{n}}{p_{n+1} \cdot p_{n}} .
$$

Finally we fix the remaining freedom with the constraint we just ignored: imposing $p_{1}$ hard with $\delta(n, a, b)$. This choice mixes the two terms

$$
\mathcal{O}\left(z^{-3}\right) \sim B_{n+1} \rightarrow A_{n}\left(z p_{1}\right)\left(\frac{a_{1}}{z p_{n+1} \cdot p_{1}}-\frac{a_{n}}{z p_{n+1} \cdot p_{1}}\right) .
$$

Note that 1 and $n$ are nonadjacent at $(n+1)$-points, so we demand $B_{n+1}$ scale as $\mathcal{O}\left(z^{-3}\right)$, whereas by assumption $A_{n}$ scales as $\mathcal{O}\left(z^{-1}\right)$ since 1 and $n$ are adjacent at $n$-points. This implies we must have $a_{1}=a_{n}$, finally fixing the full leading soft theorem for $B_{n+1}$, and hence $B_{n+1}=A_{n+1}$, completing the induction.

\section{APPENDIX B: BI CONTACT TERM DOUBLE SOFT SCALING}

We will prove there is no polynomial of mass dimension [n] with double soft scaling $\mathcal{O}\left(\tau^{3}\right)$. Taking a double soft limit in $p_{n+1}=q$ and $p_{n+2}=q$ (which by assumption must start at order $\tau^{3}$ ):

$$
\begin{aligned}
C_{n+2}\left(p^{n+2}\right)= & \tau^{3}\left(q^{\mu} q \cdot p C_{n}^{\mu}\left(p^{n-1}\right)\right. \\
& \left.+q^{\mu} q^{\nu} q^{\rho} C_{n}^{\mu \nu \rho}\left(p^{n-1}\right)+\ldots\right) .
\end{aligned}
$$

Next we impose the other double soft limits. In fact, we will not impose any double soft limit involving particle $n$, which we remove via momentum conservation. This is a stronger statement to prove, but what it buys us is now we do not need to worry about cross term cancellations. Therefore now we need to show there are no tensor polynomials $C_{n}^{\mu}\left(p^{n-1}\right)$ or $C_{n}^{\mu \nu \rho}\left(p^{n-1}\right)$ with $\mathcal{O}\left(\tau^{3}\right)$ scaling. We can keep repeating the argument until we end up needing to show that a "totally tensorized" polynomial

$$
C_{n}^{\mu_{1} \ldots \mu_{k}}\left(p^{k}\right)
$$

cannot have an enhanced scaling in arbitrarily high dimensions, which is obvious, as there can be no nontrivial cancellations between terms of this tensor.
[1] N. Arkani-Hamed, L. Rodina, and J. Trnka, Locality and Unitarity of Scattering Amplitudes from Singularities and Gauge Invariance, Phys. Rev. Lett. 120, 231602 (2018).

[2] L. Rodina, Uniqueness from gauge invariance and the Adler zero, J. High Energy Phys. 09 (2019) 084.

[3] L. Susskind and G. Frye, Algebraic aspects of pionic duality diagrams, Phys. Rev. D 1, 1682 (1970).

[4] H. Osborn, Implications of adler zeros for multipion processes, Lett. Nuovo Cimento 2, 717 (1969).

[5] S. L. Adler, Consistency conditions on the strong interactions implied by a partially conserved axial vector current, Phys. Rev. 137, B1022 (1965).

[6] J. R. Ellis and B. Renner, On the relationship between chiral and dual models, Nucl. Phys. B21, 205 (1970).

[7] N. Arkani-Hamed, F. Cachazo, and J. Kaplan, What is the simplest quantum field theory?, J. High Energy Phys. 09 (2010) 016.
[8] C. Cheung, K. Kampf, J. Novotny, and J. Trnka, Effective Field Theories from Soft Limits of Scattering Amplitudes, Phys. Rev. Lett. 114, 221602 (2015).

[9] C. Cheung, K. Kampf, J. Novotny, C.-H. Shen, and J. Trnka, A periodic table of effective field theories, J. High Energy Phys. 02 (2017) 020.

[10] F. Cachazo and A. Strominger, Evidence for a new soft graviton theorem, arXiv:1404.4091.

[11] A. Strominger and A. Zhiboedov, Gravitational memory, BMS supertranslations and soft theorems, J. High Energy Phys. 01 (2016) 086.

[12] T. He, V. Lysov, P. Mitra, and A. Strominger, BMS supertranslations and Weinberg's soft graviton theorem, J. High Energy Phys. 05 (2015) 151.

[13] S. W. Hawking, M. J. Perry, and A. Strominger, Soft Hair on Black Holes, Phys. Rev. Lett. 116, 231301 (2016). 
[14] L. Rodina, Scattering Amplitudes from Soft Theorems and Infrared Behavior, Phys. Rev. Lett. 122, 071601 (2019).

[15] R. Britto, F. Cachazo, B. Feng, and E. Witten, Direct Proof of Tree-Level Recursion Relation in Yang-Mills Theory, Phys. Rev. Lett. 94, 181602 (2005).

[16] P. Benincasa and F. Cachazo, Consistency conditions on the S-matrix of massless particles, arXiv:0705.4305.

[17] D. A. McGady and L. Rodina, Higher-spin massless $S$-matrices in four-dimensions, Phys. Rev. D 90, 084048 (2014).

[18] K. Kampf, J. Novotny, and J. Trnka, Tree-level amplitudes in the nonlinear sigma model, J. High Energy Phys. 05 (2013) 032.

[19] K. Kampf, J. Novotny, and J. Trnka, Recursion relations for tree-level amplitudes in the $S U(N)$ nonlinear sigma model, Phys. Rev. D 87, 081701 (2013).

[20] C. Cheung, C.-H. Shen, and J. Trnka, Simple recursion relations for general field theories, J. High Energy Phys. 06 (2015) 118.

[21] H. Luo and C. Wen, Recursion relations from soft theorems, J. High Energy Phys. 03 (2016) 088.

[22] F. Cachazo, P. Cha, and S. Mizera, Extensions of theories from soft limits, J. High Energy Phys. 06 (2016) 170.

[23] I. Low and Z. Yin, Soft bootstrap and effective field theories, arXiv:1904.12859.

[24] H. Elvang, M. Hadjiantonis, C. R. T. Jones, and S. Paranjape, Soft bootstrap and supersymmetry, J. High Energy Phys. 01 (2019) 195.

[25] N. Arkani-Hamed and J. Kaplan, On tree amplitudes in gauge theory and gravity, J. High Energy Phys. 04 (2008) 076.

[26] L. Rodina, Uniqueness from locality and BCFW shifts, J. High Energy Phys. 09 (2019) 078.

[27] Z. Bern, J. J. M. Carrasco, and H. Johansson, New relations for gauge-theory amplitudes, Phys. Rev. D 78, 085011 (2008).

[28] Z. Bern, J. J. M. Carrasco, and H. Johansson, Perturbative Quantum Gravity as a Double Copy of Gauge Theory, Phys. Rev. Lett. 105, 061602 (2010).

[29] H. Kawai, D. C. Lewellen, and S. H. H. Tye, A relation between tree amplitudes of closed and open strings, Nucl. Phys. B269, 1 (1986).

[30] F. Cachazo, S. He, and E. Y. Yuan, Scattering equations and Kawai-Lewellen-Tye orthogonality, Phys. Rev. D 90, 065001 (2014).

[31] F. Cachazo, S. He, and E. Y. Yuan, Scattering of Massless Particles in Arbitrary Dimensions, Phys. Rev. Lett. 113, 171601 (2014).

[32] F. Cachazo, S. He, and E. Y. Yuan, Scattering of massless particles: Scalars, gluons and gravitons, J. High Energy Phys. 07 (2014) 033.

[33] F. Cachazo, S. He, and E. Y. Yuan, Einstein-Yang-Mills scattering amplitudes from scattering equations, J. High Energy Phys. 01 (2015) 121.

[34] F. Cachazo, S. He, and E. Y. Yuan, Scattering equations and matrices: From Einstein to Yang-Mills, DBI and NLSM, J. High Energy Phys. 07 (2015) 149.

[35] S. He and Y. Zhang, New formulas for amplitudes from higher-dimensional operators, J. High Energy Phys. 02 (2017) 019.
[36] C. Cheung, C.-H. Shen, and C. Wen, Unifying relations for scattering amplitudes, J. High Energy Phys. 02 (2018) 095.

[37] C. Cheung, G. N. Remmen, C.-H. Shen, and C. Wen, Pions as gluons in higher dimensions, J. High Energy Phys. 04 (2018) 129.

[38] Z. Bern, J. J. Carrasco, W.-M. Chen, H. Johansson, and R. Roiban, Gravity Amplitudes as Generalized Double Copies of Gauge-Theory Amplitudes, Phys. Rev. Lett. 118, 181602 (2017).

[39] Z. Bern, J. J. Carrasco, W.-M. Chen, A. Edison, H. Johansson, J. Parra-Martinez, R. Roiban, and M. Zeng, Ultraviolet properties of $\mathcal{N}=8$ supergravity at five loops, Phys. Rev. D 98, 086021 (2018).

[40] R. H. Boels, B. A. Kniehl, O. V. Tarasov, and G. Yang, Color-kinematic duality for form factors, J. High Energy Phys. 02 (2013) 063.

[41] G. Yang, Color-Kinematics Duality and Sudakov Form Factor at Five Loops for $N=4$ Supersymmetric YangMills Theory, Phys. Rev. Lett. 117, 271602 (2016).

[42] L. Borsten, M. J. Duff, L. J. Hughes, and S. Nagy, Magic Square from Yang-Mills Squared, Phys. Rev. Lett. 112, 131601 (2014).

[43] A. Anastasiou, L. Borsten, M. J. Duff, L. J. Hughes, and S. Nagy, Yang-Mills Origin of Gravitational Symmetries, Phys. Rev. Lett. 113, 231606 (2014).

[44] S. Nagy, Chiral squaring, J. High Energy Phys. 07 (2016) 142.

[45] A. Anastasiou, L. Borsten, M. J. Hughes, and S. Nagy, Global symmetries of Yang-Mills squared in various dimensions, J. High Energy Phys. 01 (2016) 148.

[46] G. Cardoso, S. Nagy, and S. Nampuri, Multi-centered $\mathcal{N}=2$ BPS black holes: A double copy description, J. High Energy Phys. 04 (2017) 037.

[47] G. L. Cardoso, S. Nagy, and S. Nampuri, A double copy for $\mathcal{N}=2$ supergravity: A linearised tale told on-shell, J. High Energy Phys. 10 (2016) 127.

[48] A. Anastasiou, L. Borsten, M. J. Duff, S. Nagy, and M. Zoccali, Gravity as Gauge Theory Squared: A Ghost Story, Phys. Rev. Lett. 121, 211601 (2018).

[49] D. Neill and I. Z. Rothstein, Classical space-times from the S matrix, Nucl. Phys. B877, 177 (2013).

[50] R. Monteiro, D. O'Connell, and C. D. White, Black holes and the double copy, J. High Energy Phys. 12 (2014) 056.

[51] A. Luna, R. Monteiro, D. O'Connell, and C. D. White, The classical double copy for Taub-NUT spacetime, Phys. Lett. B 750, 272 (2015).

[52] A. K. Ridgway and M. B. Wise, Static spherically symmetric Kerr-Schild metrics and implications for the classical double copy, Phys. Rev. D 94, 044023 (2016).

[53] A. Luna, R. Monteiro, I. Nicholson, D. O'Connell, and C. D. White, The double copy: Bremsstrahlung and accelerating black holes, J. High Energy Phys. 06 (2016) 023.

[54] A. Luna, R. Monteiro, I. Nicholson, A. Ochirov, D. O'Connell, N. Westerberg, and C. D. White, Perturbative spacetimes from Yang-Mills theory, J. High Energy Phys. 04 (2017) 069.

[55] C. D. White, Exact solutions for the biadjoint scalar field, Phys. Lett. B 763, 365 (2016). 
[56] A. Luna, R. Monteiro, I. Nicholson, and D. O'Connell, Type D spacetimes and the Weyl double copy, Classical Quantum Gravity 36, 065003 (2019).

[57] A. Luna, I. Nicholson, D. O'Connell, and C. D. White, Inelastic black hole scattering from charged scalar amplitudes, J. High Energy Phys. 03 (2018) 044.

[58] W. D. Goldberger, S. G. Prabhu, and J. O. Thompson, Classical gluon and graviton radiation from the biadjoint scalar double copy, Phys. Rev. D 96, 065009 (2017).

[59] W. D. Goldberger and A. K. Ridgway, Radiation and the classical double copy for color charges, Phys. Rev. D 95, 125010 (2017).

[60] W. D. Goldberger and A. K. Ridgway, Bound states and the classical double copy, Phys. Rev. D 97, 085019 (2018).

[61] M. Carrillo-González, R. Penco, and M. Trodden, The classical double copy in maximally symmetric spacetimes, J. High Energy Phys. 04 (2018) 028.

[62] M. Gurses and B. Tekin, Classical double copy: KerrSchild-Kundt metrics from Yang-Mills theory, Phys. Rev. D 98, 126017 (2018).

[63] Z. Bern, C. Cheung, R. Roiban, C.-H. Shen, M. P. Solon, and M. Zeng, Scattering Amplitudes and the Conservative Hamiltonian for Binary Systems at Third Post-Minkowskian Order, Phys. Rev. Lett. 122, 201603 (2019).

[64] Z. Bern, C. Cheung, R. Roiban, C.-H. Shen, M. P. Solon, and M. Zeng, Black hole binary dynamics from the double copy and effective theory, J. High Energy Phys. 10 (2019) 206.

[65] Z. Bern, J. J. Carrasco, M. Chiodaroli, H. Johansson, and R. Roiban, The duality between color and kinematics and its applications, arXiv:1909.01358.

[66] Z. Bern, A. De Freitas, and H. L. Wong, On the Coupling of Gravitons to Matter, Phys. Rev. Lett. 84, 3531 (2000).

[67] Y.-J. Du, B. Feng, and C.-H. Fu, BCJ relation of color scalar theory and KLT relation of gauge theory, J. High Energy Phys. 08 (2011) 129.

[68] N. E. J. Bjerrum-Bohr, P. H. Damgaard, R. Monteiro, and D. O'Connell, Algebras for amplitudes, J. High Energy Phys. 06 (2012) 061.

[69] M. Chiodaroli, M. Günaydin, H. Johansson, and R. Roiban, Scattering amplitudes in $\mathcal{N}=2$ Maxwell-Einstein and Yang-Mills/Einstein supergravity, J. High Energy Phys. 01 (2015) 081.

[70] M. Chiodaroli, M. Gunaydin, H. Johansson, and R. Roiban, Spontaneously broken Yang-Mills-Einstein supergravities as double copies, J. High Energy Phys. 06 (2017) 064.

[71] I. Low and Z. Yin, Ward Identity and Scattering Amplitudes for Nonlinear Sigma Models, Phys. Rev. Lett. 120, 061601 (2018).

[72] V. Del Duca, L. J. Dixon, and F. Maltoni, New color decompositions for gauge amplitudes at tree and loop level, Nucl. Phys. B571, 51 (2000).

[73] S. Mizera, Inverse of the string theory KLT kernel, J. High Energy Phys. 06 (2017) 084.

[74] N. E. J. Bjerrum-Bohr, P. H. Damgaard, B. Feng, and T. Sondergaard, Gravity and Yang-Mills amplitude relations, Phys. Rev. D 82, 107702 (2010).
[75] J. A. Cronin, Phenomenological model of strong and weak interactions in chiral $U(3) \times U(3)$, Phys. Rev. 161, 1483 (1967).

[76] S. Weinberg, Dynamical Approach to Current Algebra, Phys. Rev. Lett. 18, 188 (1967).

[77] S. Weinberg, Nonlinear realizations of chiral symmetry, Phys. Rev. 166, 1568 (1968).

[78] A. J. MacFarlane, A. Sudbery, and P. H. Weisz, On GellMann's $\lambda$-matrices, $d$ - and $f$-tensors, octets, and parametrizations of $s u(3)$, Commun. Math. Phys. 11, 77 (1968).

[79] J. J. M. Carrasco, C. R. Mafra, and O. Schlotterer, Abelian Z-theory: NLSM amplitudes and $\alpha^{\prime}$-corrections from the open string, J. High Energy Phys. 06 (2017) 093.

[80] I. Low and Z. Yin, The infrared structure of NambuGoldstone bosons, J. High Energy Phys. 10 (2018) 078.

[81] J. Bagger and A. Galperin, A new Goldstone multiplet for partially broken supersymmetry, Phys. Rev. D 55, 1091 (1997).

[82] E. Bergshoeff, F. Coomans, R. Kallosh, C. S. Shahbazi, and A. Van Proeyen, Dirac-Born-Infeld-Volkov-Akulov and deformation of supersymmetry, J. High Energy Phys. 08 (2013) 100.

[83] C. Cheung, K. Kampf, J. Novotny, C.-H. Shen, J. Trnka, and C. Wen, Vector Effective Field Theories from Soft Limits, Phys. Rev. Lett. 120, 261602 (2018).

[84] G. R. Dvali, G. Gabadadze, and M. Porrati, 4-D gravity on a brane in 5-D Minkowski space, Phys. Lett. B 485, 208 (2000).

[85] A. Nicolis, R. Rattazzi, and E. Trincherini, Galileon as a local modification of gravity, Phys. Rev. D 79, 064036 (2009).

[86] C. de Rham, G. Gabadadze, and A. J. Tolley, Resummation of Massive Gravity, Phys. Rev. Lett. 106, 231101 (2011).

[87] K. Kampf and J. Novotny, Unification of Galileon dualities, J. High Energy Phys. 10 (2014) 006.

[88] K. Hinterbichler and A. Joyce, Hidden symmetry of the Galileon, Phys. Rev. D 92, 023503 (2015).

[89] J. Novotny, Geometry of special Galileons, Phys. Rev. D 95, 065019 (2017).

[90] S. He, Y.-t. Huang, and C. Wen, Loop corrections to soft theorems in gauge theories and gravity, J. High Energy Phys. 12 (2014) 115.

[91] S. He, Z. Liu, and J.-B. Wu, Scattering equations, twistorstring formulas and double-soft limits in four dimensions, J. High Energy Phys. 07 (2016) 060.

[92] A. L. Guerrieri, Y.-t. Huang, Z. Li, and C. Wen, On the exactness of soft theorems, J. High Energy Phys. 12 (2017) 052 .

[93] Z. Bern, S. Davies, P. Di Vecchia, and J. Nohle, Lowenergy behavior of gluons and gravitons from gauge invariance, Phys. Rev. D 90, 084035 (2014).

[94] Z. Bern, S. Davies, and J. Nohle, On loop corrections to subleading soft behavior of gluons and gravitons, Phys. Rev. D 90, 085015 (2014).

[95] Y.-t. Huang and C. Wen, Soft theorems from anomalous symmetries, J. High Energy Phys. 12 (2015) 143.

[96] W.-M. Chen, Y.-t. Huang, and C. Wen, New Fermionic Soft Theorems for Supergravity Amplitudes, Phys. Rev. Lett. 115, 021603 (2015). 
[97] I. Low, Double soft theorems and shift symmetry in nonlinear sigma models, Phys. Rev. D 93, 045032 (2016).

[98] H. Elvang, C. R. T. Jones, and S. G. Naculich, Soft Photon and Graviton Theorems in Effective Field Theory, Phys. Rev. Lett. 118, 231601 (2017).

[99] P. Di Vecchia, R. Marotta, M. Mojaza, and J. Nohle, New soft theorems for the gravity dilaton and the NambuGoldstone dilaton at subsubleading order, Phys. Rev. D 93, 085015 (2016).

[100] P. Di Vecchia, R. Marotta, and M. Mojaza, Soft theorem for the graviton, dilaton and the Kalb-Ramond field in the bosonic string, J. High Energy Phys. 05 (2015) 137.

[101] B. U.W. Schwab and A. Volovich, Subleading Soft Theorem in Arbitrary Dimensions from Scattering Equations, Phys. Rev. Lett. 113, 101601 (2014).

[102] A. Strominger, Lectures on the infrared structure of gravity and gauge theory, arXiv:1703.05448.

[103] F. Cachazo, S. He, and E. Y. Yuan, New double soft emission theorems, Phys. Rev. D 92, 065030 (2015).

[104] S. He and Q. Yang, An etude on recursion relations and triangulations, J. High Energy Phys. 05 (2019) 040.

[105] P. Benincasa, C. Boucher-Veronneau, and F. Cachazo, Taming tree amplitudes in general relativity, J. High Energy Phys. 11 (2007) 057.

[106] P. C. Schuster and N. Toro, Constructing the tree-level Yang-Mills S-matrix using complex factorization, J. High Energy Phys. 06 (2009) 079.

[107] R. H. Boels and R. Medina, Graviton and Gluon Scattering from First Principles, Phys. Rev. Lett. 118, 061602 (2017).

[108] F. Cachazo, P. Svrcek, and E. Witten, MHV vertices and tree amplitudes in gauge theory, J. High Energy Phys. 09 (2004) 006.

[109] F. A. Berends and W. T. Giele, Recursive calculations for processes with $n$ gluons, Nucl. Phys. B306, 759 (1988).

[110] S. Lee, C. R. Mafra, and O. Schlotterer, Non-linear gauge transformations in $D=10 \mathrm{SYM}$ theory and the BCJ duality, J. High Energy Phys. 03 (2016) 090.

[111] C. R. Mafra, Berends-Giele recursion for double-colorordered amplitudes, J. High Energy Phys. 07 (2016) 080.

[112] S. Mizera and B. Skrzypek, Perturbiner methods for effective field theories and the double copy, J. High Energy Phys. 10 (2018) 018.

[113] J. Broedel, M. de Leeuw, J. Plefka, and M. Rosso, Constraining subleading soft gluon and graviton theorems, Phys. Rev. D 90, 065024 (2014).

[114] M. Froissart, Asymptotic behavior and subtractions in the Mandelstam representation, Phys. Rev. 123, 1053 (1961).

[115] R. Kleiss and H. Kuijf, Multi-gluon cross-sections and five jet production at hadron colliders, Nucl. Phys. B312, 616 (1989).

[116] G. Chen and Y.-J. Du, Amplitude relations in nonlinear sigma model, J. High Energy Phys. 01 (2014) 061.
[117] B. Feng, R. Huang, and Y. Jia, Gauge amplitude identities by on-shell recursion relation in S-matrix program, Phys. Lett. B 695, 350 (2011).

[118] J. J. M. Carrasco, C. R. Mafra, and O. Schlotterer, Semiabelian Z-theory: NLSM $+\phi^{3}$ from the open string, J. High Energy Phys. 08 (2017) 135.

[119] S. Caron-Huot, Z. Komargodski, A. Sever, and A. Zhiboedov, Strings from massive higher spins: The asymptotic uniqueness of the Veneziano amplitude, J. High Energy Phys. 10 (2017) 026.

[120] D. A. McGady and L. Rodina, Recursion relations for graviton scattering amplitudes from Bose symmetry and bonus scaling laws, Phys. Rev. D 91, 105010 (2015).

[121] Z. Bern, T. Dennen, Y.-t. Huang, and M. Kiermaier, Gravity as the square of gauge theory, Phys. Rev. D 82, 065003 (2010).

[122] R. H. Boels and R.S. Isermann, On powercounting in perturbative quantum gravity theories through colorkinematic duality, J. High Energy Phys. 06 (2013) 017.

[123] N. Arkani-Hamed and J. Trnka, The amplituhedron, J. High Energy Phys. 10 (2014) 030.

[124] N. Arkani-Hamed, Y. Bai, S. He, and G. Yan, Scattering forms and the positive geometry of kinematics, color and the worldsheet, J. High Energy Phys. 05 (2018) 096.

[125] F. Loebbert, M. Mojaza, and J. Plefka, Hidden conformal symmetry in tree-level graviton scattering, J. High Energy Phys. 05 (2018) 208.

[126] J. L. Bourjaily, E. Herrmann, and J. Trnka, Maximally supersymmetric amplitudes at infinite loop momentum, Phys. Rev. D 99, 066006 (2019).

[127] S. Caron-Huot and M. Wilhelm, Renormalization group coefficients and the S-matrix, J. High Energy Phys. 12 (2016) 010.

[128] S. Pasterski and S.-H. Shao, Conformal basis for flat space amplitudes, Phys. Rev. D 96, 065022 (2017).

[129] S. Pasterski, S.-H. Shao, and A. Strominger, Flat space amplitudes and conformal symmetry of the celestial sphere, Phys. Rev. D 96, 065026 (2017).

[130] A. Schreiber, A. Volovich, and M. Zlotnikov, Tree-level gluon amplitudes on the celestial sphere, Phys. Lett. B 781, 349 (2018).

[131] L. Donnay, A. Puhm, and A. Strominger, Conformally soft photons and gravitons, J. High Energy Phys. 01 (2019) 184.

[132] D. Nandan, A. Schreiber, A. Volovich, and M. Zlotnikov, Celestial amplitudes: Conformal partial waves and soft limits, J. High Energy Phys. 10 (2019) 018.

[133] W. Fan, A. Fotopoulos, and T. R. Taylor, Soft limits of Yang-Mills amplitudes and conformal correlators, J. High Energy Phys. 05 (2019) 121.

[134] A. Guevara, Notes on conformal soft theorems and recursion relations in gravity, arXiv:1906.07810.

[135] S. Stieberger and T. R. Taylor, Strings on celestial sphere, Nucl. Phys. B935, 388 (2018). 